Sharif University of Technology
Scientia Iranica
Transactions E: Industrial Engineering
hCIENTIA

\title{
New operations for interval-valued Pythagorean fuzzy
}

\section{set}

\author{
X. Peng* \\ School of Information Science and Engineering, Shaoguan University, Shaoguan, People's Republic of China.
}

Received 6 September 2017; received in revised form 11 November 2017; accepted 27 January 2018

\author{
KEYWORDS \\ Interval-valued \\ Pythagorean fuzzy \\ set; \\ Interval-valued \\ Pythagorean fuzzy \\ operators; \\ Multi-criteria decision \\ making.
}

\begin{abstract}
Interval-Valued Pythagorean Fuzzy Set (IVPFS), originally proposed by Peng and Yang, is a novel tool to deal with vagueness and incertitude. As a generalized set, IVPFS has a close relationship with Interval-Valued Intuitionistic Fuzzy Set (IVIFS). IVPFS can be reduced to IVIFS, satisfying the condition $\mu^{+}+\nu^{+} \leq 1$. However, the related operations of IVPFS do not take different conditions into consideration. In this paper, we initiate some new interval-valued Pythagorean fuzzy operators $(\diamond, \square, \mathbf{\phi}, \boldsymbol{\phi}, \boldsymbol{\Psi}, \rightarrow, \$)$ and discuss their properties in relation with some existing operators $(\cup, \cap, \oplus, \otimes)$ in detail. It will promote the development of interval-valued Pythagorean fuzzy operators. Later, an algorithm is proposed to deal with Multi-Criteria Decision-Making (MCDM) problem based on the proposed operator. Finally, the effectiveness and feasibility of the proposed algorithm is demonstrated by mine emergency decision-making example.
\end{abstract}

(C) 2019 Sharif University of Technology. All rights reserved.

\section{Introduction}

Intuitionistic Fuzzy Set (IFS), initiated by Atanassov [1], is an extension of fuzzy set theory [2]. IFS is characterized by a membership degree and a nonmembership degree and, hence, can depict the fuzzy character of data more comprehensively and detailedly. The prominent characteristic of IFS is that it assigns a membership degree and a nonmembership degree to each element with their sum equal to or less than one. However, in some practical decision-making processes, the sum of the membership degree and the nonmembership degree to which an alternative that satisfies the expert recommended criterion is provided, may be larger than one; however, their square sum is equal to or less than one.

Hence, Yager [3] examined Pythagorean Fuzzy Set (PFS) characterized by a membership degree and a nonmembership degree that satisfies the case in which

\footnotetext{
*. E-mail address: 952518336@qq.com.
}

doi: $10.24200 /$ sci. 2018.5142.1119 the square sum of its membership degree and nonmembership degree is less than or equal to one. Yager and Abbasov [4] gave an example to illustrate this situation: an expert giving his support for membership of an alternative is $\frac{\sqrt{3}}{2}$ and his nonmembership is $\frac{1}{2}$. Since the sum of the two values is bigger than 1 , they are not available for IFS, yet are feasible for PFS because:

$$
\left(\frac{\sqrt{3}}{2}\right)^{2}+\left(\frac{1}{2}\right)^{2} \leq 1
$$

Obviously, PFS is more effective than IFS in modeling the vagueness of the practical Multi-Criteria DecisionMaking (MCDM) problems.

The PFS has been investigated from different perspectives, including decision-making technologies [513], aggregation operators [14-22], information measures [23-25], the extensions of PFS [26-31], and fundamental properties [32-34]. In particular, an extension of PFS, named Interval-Valued Pythagorean Fuzzy Set (IVPFS) [10,21], is a hot topic at present [35].

Peng and Yang [21] proposed some new operations $(\cup, \cap, \oplus, \otimes)$ for IVPFS and discussed their properties 
in detail. Meanwhile, they studied two interval-valued Pythagorean fuzzy aggregation operators for integrating the interval-valued Pythagorean fuzzy information, such as IVPFWA and IVPFWG operators, and presented an interval-valued Pythagorean fuzzy elimination and choice translating reality method (ELECTRE) to solve Multi-Criteria Group Decision Making (MCGDM) problem with uncertainty. Liang et al. [17] conceived the maximizing deviation method based on interval-valued Pythagorean fuzzy weighted aggregating operator for MCGDM problem. Garg [36] introduced a novel interval-valued Pythagorean fuzzy accuracy function for solving MCDM problem. Rahman et al. [37] discussed interval-valued Pythagorean fuzzy geometric aggregation operators and their application to MCGDM problem. Garg [38] proposed a new improved score function of an interval-valued Pythagorean fuzzy set-based TOPSIS method. Chen [39] pioneered the IVPF outranking algorithm with a closeness-based assignment model for MCDM.

In order to enrich the operations of IVPFS, we define some new interval-valued Pythagorean fuzzy operators $(\diamond, \square, \mathbf{\$}, \boldsymbol{\$}, \rightarrow)$ and discuss their properties with some existing operators $(\cup, \cap, \oplus, \otimes)$ in detail.

To facilitate our discussion, the remainder of this paper is organized as follows. In Section 2, we review some fundamental conceptions of IVIFS and IVPFS. In Section 3, we propose some new operations for IVPFS and present some interesting properties. Meanwhile, some new operations and the existing operators are compared in detail. In Section 4, a new decisionmaking method based on operator is proposed and a comparison is constructed. The paper is concluded in Section 5 .

\section{Preliminaries}

This section presents the basic notions, definitions, and properties of IVIFS and IVPFS.

Definition 1. [40] Let $\operatorname{Int}([0,1])$ denote the set of all closed subintervals of $[0,1]$. Let $X$ be a universe of discourse. An IVIFS $\widetilde{I}$ in $X$ is given by:

$$
\widetilde{I}=\left\{<x, \mu_{\widetilde{I}}(x), \nu_{\widetilde{I}}(x)>\mid x \in X\right\},
$$

where the functions $\mu_{\tilde{I}}: X \rightarrow \operatorname{Int}([0,1])(x \in X \rightarrow$ $\left.\mu_{\widetilde{I}}(x) \subseteq[0,1]\right)$ and $\nu_{\widetilde{I}}: X \rightarrow \operatorname{Int}([0,1])(x \in X \rightarrow$ $\left.\nu_{\widetilde{I}}(x) \subseteq[0,1]\right)$ denote the membership degree and nonmembership degree of the element $x \in X$ to set $\widetilde{I}$, respectively, and for every $x \in X, 0 \leq \sup \left\{\mu_{\tilde{I}}(x)\right\}+$ $\sup \left\{\nu_{\widetilde{T}}(x)\right\} \leq 1$. Also, for each $x \in X, \mu_{\widetilde{I}}(x)$ and $\nu_{\widetilde{I}}(x)$ are closed intervals and their lower and upper bounds are denoted by $\mu_{\widetilde{I}}^{-}(x), \mu_{\widetilde{I}}^{+}(x), \nu_{\widetilde{I}}^{-}(x), \nu_{\widetilde{I}}^{+}(x)$, respectively. Therefore, $\widetilde{I}$ can also express another style as follows:

$$
\widetilde{I}=\left\{<x,\left[\mu_{\widetilde{I}}^{-}(x), \mu_{\widetilde{I}}^{+}(x)\right],\left[\nu_{\widetilde{I}}^{-}(x), \nu_{\widetilde{I}}^{+}(x)\right]>\mid x \in X\right\},
$$

whose expression is subject to the condition $0 \leq$ $\mu_{\widetilde{I}}^{+}(x)+\nu_{\widetilde{I}}^{+}(x) \leq 1$. The degree of indeterminacy $\pi_{\widetilde{I}}(x)=\left[\pi_{\widetilde{I}}^{-}(x), \pi_{\widetilde{I}}^{+}(x)\right]=\left[1-\mu_{\widetilde{I}}^{+}(x)-\nu_{\widetilde{I}}^{+}(x), 1-\right.$ $\left.\mu_{\tilde{I}}^{-}(x)-\nu_{\widetilde{I}}^{-}(x)\right]$. For convenience, Xu [41] called $\tilde{i}=\left(\left[\mu_{\tilde{i}}^{-}, \mu_{\tilde{i}}^{+}\right]\left[\nu_{\tilde{i}}^{-}, \nu_{\tilde{i}}^{+}\right]\right)$an Interval-Valued Intuitionistic Fuzzy Number (IVIFN).

Definition 2. [10] Let $\operatorname{Int}([0,1])$ denote the set of all closed subintervals of $[0,1]$, and $X$ be a universe of discourse. An IVPFS $\widetilde{P}$ in $X$ is given by:

$$
\widetilde{P}=\left\{<x, \mu_{\widetilde{P}}(x), \nu_{\widetilde{P}}(x)>\mid x \in X\right\},
$$

whose functions $\mu_{\widetilde{P}}: X \rightarrow \operatorname{Int}([0,1])(x \in X \rightarrow$ $\left.\mu_{\widetilde{P}}(x) \subseteq[0,1]\right)$ and $\nu_{\widetilde{P}}: X \rightarrow \operatorname{Int}([0,1])(x \in X \rightarrow$ $\left.\nu_{\widetilde{P}}(x) \subseteq[0,1]\right)$ denote the membership degree and nonmembership degree of the element $x \in X$ to the set $\widetilde{P}$, respectively, and for every $x \in X, 0 \leq \sup \left\{\left(\mu_{\widetilde{P}}(x)\right)^{2}\right\}+$ $\sup \left\{\left(\mu_{\widetilde{P}}(x)\right)^{2}\right\} \leq 1$. Also, for each $x \in X, \mu_{\widetilde{P}}(x)$ and $\nu_{\widetilde{P}}(x)$ are closed intervals and their lower and upper bounds are denoted by $\mu_{\widetilde{P}}^{-}(x), \mu_{\widetilde{P}}^{+}(x), \nu_{\widetilde{P}}^{-}(x)$, and $\nu_{\widetilde{P}}^{+}(x)$, respectively. So, $\widetilde{P}$ can also be expressed in another style as follows:

$$
\widetilde{P}=\left\{<x,\left[\mu_{\widetilde{P}}^{-}(x), \mu_{\widetilde{P}}^{+}(x)\right],\left[\nu_{\widetilde{P}}^{-}(x), \nu_{\widetilde{P}}^{+}(x)\right]>\mid x \in X\right\},(4)
$$

whose expression is subject to the condition $0 \leq$ $\left(\mu_{\widetilde{P}}^{+}(x)\right)^{2}+\left(\nu_{\widetilde{P}}^{+}(x)\right)^{2} \leq 1$. The degree of indeterminacy $\pi_{P}(x)$ is shown as follows:

$$
\begin{aligned}
\pi_{\widetilde{P}}(x)= & {\left[\pi_{\widetilde{P}}^{-}(x), \pi_{\widetilde{P}}^{+}(x)\right] } \\
= & {\left[\sqrt{1-\left(\mu_{\widetilde{P}}^{+}(x)\right)^{2}-\left(\nu_{\widetilde{P}}^{+}(x)\right)^{2}},\right.} \\
& \left.\sqrt{1-\left(\mu_{\widetilde{P}}^{-}(x)\right)^{2}-\left(\nu_{\widetilde{P}}^{-}(x)\right)^{2}}\right] .
\end{aligned}
$$

For convenience, Zhang [10] called:

$$
\widetilde{p}=\left(\left[\mu_{\widetilde{p}}^{-}, \mu_{\widetilde{p}}^{+}\right],\left[\nu_{\widetilde{p}}^{-}, \nu_{\widetilde{p}}^{+}\right]\right),
$$

an Interval-Valued Pythagorean Fuzzy Number (IVPFN). It is easily known that IVPFS reduces to PFS when the boundary is the same.

The main difference between IVPFNs and IVIFNs is their corresponding constraint cases, as easily shown in Figure 1 [10].

\section{Definition 3. [21] Let:}

$$
\widetilde{p}_{1}=\left(\left[\mu_{1}^{-}, \mu_{1}^{+}\right],\left[\nu_{1}^{-}, \nu_{1}^{+}\right]\right),
$$

and: 


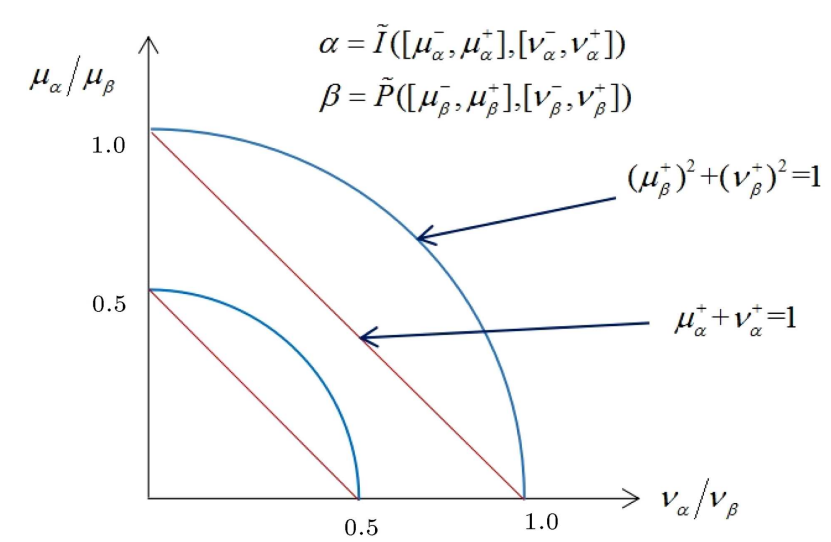

Figure 1. Comparison of spaces of the IVPFNs and IVIFNs.

$$
\widetilde{p}_{2}=\left(\left[\mu_{2}^{-}, \mu_{2}^{+}\right],\left[\nu_{2}^{-}, \nu_{2}^{+}\right]\right)
$$

be two IVPFNs, and then their relations are defined as follows:

1. $\widetilde{p}_{1}=\widetilde{p}_{2}$ iff $\mu_{1}^{-}=\mu_{2}^{-}, \mu_{1}^{+}=\mu_{2}^{+}, \nu_{1}^{-}=\nu_{2}^{-}$, and $\nu_{1}^{+}=$ $\nu_{2}^{+}$

2. $\widetilde{p}_{1} \prec \widetilde{p}_{2}$ iff $\mu_{1}^{-} \leq \mu_{2}^{-}, \mu_{1}^{+} \leq \mu_{2}^{+}, \nu_{1}^{-} \geq \nu_{2}^{-}$, and $\nu_{1}^{+} \geq$ $\nu_{2}^{+}$.

Definition 4. [21] For any IVPFN $\tilde{p}=\left(\left[\mu_{\widetilde{p}}^{-}, \mu_{\widetilde{p}}^{+}\right]\right.$, $\left.\left[\nu_{\widetilde{p}}^{-}, \nu_{\widetilde{p}}^{+}\right]\right)$, the score function of $\tilde{p}$ is defined as follows:

$$
\begin{aligned}
& s(\widetilde{p})=\frac{1}{2}\left[\left(\mu_{\tilde{p}}^{-}\right)^{2}+\left(\mu_{\widetilde{p}}^{+}\right)^{2}-\left(\nu_{\widetilde{p}}^{-}\right)^{2}-\left(\nu_{\widetilde{p}}^{+}\right)^{2}\right], \\
& s(\widetilde{p}) \in[-1,1] .
\end{aligned}
$$

Definition 5. [21] For any IVPFN $\tilde{p}=\left(\left[\mu_{\tilde{p}}^{-}, \mu_{\tilde{p}}^{+}\right]\right.$, $\left.\left[\nu_{\widetilde{p}}^{-}, \nu_{\widetilde{p}}^{+}\right]\right)$, the accuracy function of $\widetilde{p}$ is defined as follows:

$$
\begin{aligned}
& a(\tilde{p})=\frac{1}{2}\left[\left(\mu_{\tilde{p}}^{-}\right)^{2}+\left(\mu_{\widetilde{p}}^{+}\right)^{2}+\left(\nu_{\widetilde{p}}^{-}\right)^{2}+\left(\nu_{\widetilde{p}}^{+}\right)^{2}\right], \\
& a(\widetilde{p}) \in[0,1] .
\end{aligned}
$$

For any two IVPFNs, $\widetilde{p}_{1}, \widetilde{p}_{2}$, the comparison rule is defined as follows:

1. if $s\left(\widetilde{p}_{1}\right)>s\left(\widetilde{p}_{2}\right)$, then $\widetilde{p}_{1} \succ \widetilde{p}_{2}$;

2. if $s\left(\widetilde{p}_{1}\right)=s\left(\widetilde{p}_{2}\right)$, then:
(a) if $a\left(\tilde{p}_{1}\right)>a\left(\tilde{p}_{2}\right)$, then $\widetilde{p}_{1} \succ \widetilde{p}_{2}$;
(b) if $a\left(\widetilde{p}_{1}\right)=a\left(\tilde{p}_{2}\right)$, then $\widetilde{p}_{1}=\widetilde{p}_{2}$.

\section{Interval-valued Pythagorean fuzzy operators}

This section reviews some existing interval-valued Pythagorean fuzzy operators and proposes some new interval-valued Pythagorean fuzzy operators.

\subsection{Some existing interval-valued Pythagorean fuzzy operators}

Definition 6. [21] Let $\widetilde{P}, \widetilde{P}_{1}$, and $\widetilde{P}_{2}$, be three IVPFSs, and $\lambda>0$. Then, their operations are defined as follows:

1. $\widetilde{P}_{1} \cup \widetilde{P}_{2}=\left\{<x,\left[\max \left\{\mu_{1}^{-}(x), \mu_{2}^{-}(x)\right\}, \max \left\{\mu_{1}^{+}(x)\right.\right.\right.$, $\left.\left.\mu_{2}^{+}(x)\right\}\right],\left[\min \left\{\nu_{1}^{-}(x), \nu_{2}^{-}(x)\right\}, \min \left\{\nu_{1}^{+}(x), \nu_{2}^{+}(x)\right\}\right]$ $>\mid x \in X\}$;

2. $\widetilde{P}_{1} \cap \widetilde{P}_{2}=\left\{<x,\left[\min \left\{\mu_{1}^{-}(x), \mu_{2}^{-}(x)\right\}, \min \left\{\mu_{1}^{+}(x)\right.\right.\right.$, $\left.\left.\mu_{2}^{+}(x)\right\}\right],\left[\max \left\{\nu_{1}^{-}(x), \nu_{2}^{-}(x)\right\}, \max \left\{\nu_{1}^{+}(x), \nu_{2}^{+}(x)\right\}\right]$ $>\mid x \in X\}$;

3. $\widetilde{P}_{1} \oplus \widetilde{P}_{2}=\{<x$, $\left[\sqrt{\left(\mu_{1}^{-}(x)\right)^{2}+\left(\mu_{2}^{-}(x)\right)^{2}-\left(\mu_{1}^{-}(x)\right)^{2}\left(\mu_{2}^{-}(x)\right)^{2}}\right.$,
$\left.\sqrt{\left(\mu_{1}^{+}(x)\right)^{2}+\left(\mu_{2}^{+}(x)\right)^{2}-\left(\mu_{1}^{+}(x)\right)^{2}\left(\mu_{2}^{+}(x)\right)^{2}}\right]$,
$\left.\left[\nu_{1}^{-}(x) \nu_{2}^{-}(x), \nu_{1}^{+}(x) \nu_{2}^{+}(x)\right]>\mid x \in X\right\} ;$

4. $\widetilde{P}_{1} \otimes \widetilde{P}_{2}=\left\{<x,\left[\mu_{1}^{-}(x) \mu_{2}^{-}(x), \mu_{1}^{+}(x) \mu_{2}^{+}(x)\right]\right.$, $\left[\sqrt{\left(\nu_{1}^{-}(x)\right)^{2}+\left(\nu_{2}^{-}(x)\right)^{2}-\left(\nu_{1}^{-}(x)\right)^{2}\left(\nu_{2}^{-}(x)\right)^{2}}\right.$, $\left.\left.\sqrt{\left(\nu_{1}^{+}(x)\right)^{2}+\left(\nu_{2}^{+}(x)\right)^{2}-\left(\nu_{1}^{+}(x)\right)^{2}\left(\nu_{2}^{+}(x)\right)^{2}}\right] \mid x \in X\right\}$;

5. $\lambda \widetilde{P}=\left\{<x,\left[\sqrt{1-\left(1-\left(\mu_{P}^{-}(x)\right)^{2}\right)^{\lambda}}\right.\right.$, $\left.\sqrt{1-\left(1-\left(\mu_{P}^{+}(x)\right)^{2}\right)^{\lambda}}\right],\left[\left(\nu_{P}^{-}(x)\right)^{\lambda},\left(\nu_{P}^{+}(x)\right)^{\lambda}\right]$ $>\mid x \in X\}$;

6. $\widetilde{P}^{\lambda}=\left\{<x,\left[\left(\mu_{P}^{-}(x)\right)^{\lambda},\left(\mu_{P}^{+}(x)\right)^{\lambda}\right]\right.$, $\left[\sqrt{1-\left(1-\left(\nu_{P}^{-}(x)\right)^{2}\right)^{\lambda}}, \sqrt{1-\left(1-\left(\nu_{P}^{+}(x)\right)^{2}\right)^{\lambda}}\right] \mid$ $x \in X\}$;

7. $P_{1} \subseteq P_{2}$ iff $\mu_{1}^{-}(x) \leq \mu_{2}^{-}(x), \mu_{1}^{+}(x) \leq \mu_{2}^{+}(x)$, $\nu_{1}^{-}(x) \geq \nu_{2}^{-}(x), \nu_{1}^{+}(x) \geq \nu_{2}^{+}(x)$, for $\forall x \in X$;

8. $P^{c}=\left\{<x,\left[\nu_{P}^{-}(x), \nu_{P}^{+}(x)\right],\left[\mu_{P}^{-}(x), \mu_{P}^{+}(x)\right]>\mid x \in\right.$ $X\}$.

\subsection{Some new interval-valued Pythagorean fuzzy operators}

Definition 7. The necessity operation on an IVPFS $P$ is denoted by $\square P$ and is defined as: $\square P=\left\{\left\langle x,\left[\mu_{P}^{-}(x), \mu_{P}^{+}(x)\right]\right.\right.$,

$$
\left.\left.\left[\sqrt{1-\left(\mu_{P}^{+}(x)\right)^{2}}, \sqrt{1-\left(\mu_{P}^{-}(x)\right)^{2}}\right]\right\rangle \mid x \in X\right\} .
$$


Example 1. Let $P$ be an IVPFS over $X$ such that:

$$
\begin{aligned}
P=\{ & <x_{1},[0.2,0.3],[0.4,0.6]>, \\
& <x_{2},[0.2,0.4],[0.5,0.8]>, \\
& <x_{3},[0.4,0.5],[0.6,0.7]>, \\
& \left.<x_{4},[0.5,0.6],[0.7,0.8]>\right\} .
\end{aligned}
$$

Then, we can easily compute $\square P$ by the above definition shown as follows:

$$
\begin{aligned}
\square P=\{ & <x_{1},[0.2,0.3],[0.9539,0.9798]>, \\
& <x_{2},[0.2,0.4],[0.9165,0.9798]>, \\
& <x_{3},[0.4,0.5],[0.8660,0.9165]>, \\
& \left.<x_{4},[0.5,0.6],[0.8,0.8660]>\right\} .
\end{aligned}
$$

Definition 8. The possibility operation on an IVPFS $P$ is denoted by $\diamond P$ and is defined as:

$$
\begin{aligned}
\diamond P=\{ & <x,\left[\sqrt{1-\left(\nu_{P}^{+}(x)\right)^{2}}, \sqrt{1-\left(\nu_{P}^{-}(x)\right)^{2}}\right], \\
& {\left.\left[\nu_{P}^{-}(x), \nu_{P}^{+}(x)\right]>\mid x \in X\right\} . }
\end{aligned}
$$

Example 2. If we continue to use Example 1, then we can easily compute $\diamond P$ by above definition shown as follows:

$$
\begin{aligned}
\diamond P=\{ & <x_{1},[0.2,0.3],[0.8000,0.9165]>, \\
& <x_{2},[0.2,0.4],[0.6000,0.8660]>, \\
& <x_{3},[0.4,0.5],[0.7141,0.8000]>, \\
& \left.<x_{4},[0.5,0.6],[0.6000,0.7141]>\right\} .
\end{aligned}
$$

Two new relations are defined by $P \subseteq_{\square} Q$ iff $\mu_{P}^{-}(x) \leq$ $\mu_{Q}^{-}(x), \mu_{P}^{+}(x) \leq \mu_{Q}^{+}(x)$ for $\forall x \in X$ and $P \subseteq \diamond Q$ iff $\nu_{P}^{-}(x) \geq \nu_{Q}^{-}(x), \nu_{P}^{+}(x) \geq \nu_{Q}^{+}(x)$ for $\forall x \in X$.

Definition 9. Let $P$ and $Q$ be two IVPFSs. For two IVPFSs $P$ and $Q$, the operations are defined as follows:

1. $P \mathbb{Q} Q=$

$$
\begin{aligned}
& \left\{\left\langlex,\left[\sqrt{\frac{\left(\mu_{P}^{-}(x)\right)^{2}+\left(\mu_{Q}^{-}(x)\right)^{2}}{2}}, \sqrt{\frac{\left(\mu_{P}^{+}(x)\right)^{2}+\left(\mu_{Q}^{+}(x)\right)^{2}}{2}}\right],\right.\right. \\
& \left.\left.\left[\sqrt{\frac{\left(\nu_{P}^{-}(x)\right)^{2}+\left(\nu_{Q}^{-}(x)\right)^{2}}{2}}, \sqrt{\frac{\left(\nu_{P}^{+}(x)\right)^{2}+\left(\nu_{Q}^{+}(x)\right)^{2}}{2}}\right]\right\rangle \mid x \in X\right\} ;
\end{aligned}
$$

2. $P \rightarrow Q=$

$$
\begin{aligned}
& \left\{\left\langlex,\left[\max \left\{\nu_{P}^{-}(x), \mu_{Q}^{-}(x)\right\}, \max \left\{\nu_{P}^{+}(x), \mu_{Q}^{+}(x)\right\}\right],\right.\right. \\
& \left.\left[\min \left\{\mu_{P}^{-}(x), \nu_{Q}^{-}(x)\right\}, \min \left\{\mu_{P}^{+}(x), \nu_{Q}^{+}(x)\right\}\right]\right\rangle \mid x \in \\
& X\} ;
\end{aligned}
$$

3. $P \$ Q=$

$$
\begin{aligned}
& \left\{<x,\left[\sqrt{\mu_{P}^{-}(x) \mu_{Q}^{-}(x)}, \sqrt{\mu_{P}^{+}(x) \mu_{Q}^{+}(x)}\right],\right. \\
& \left.\left[\sqrt{\nu_{P}^{-}(x) \nu_{Q}^{-}(x)}, \sqrt{\nu_{P}^{+}(x) \nu_{Q}^{+}(x)}\right]>\mid x \in X\right\} ;
\end{aligned}
$$

4. $P$ \& $Q=$

$$
\begin{aligned}
& \left\{\left\langlex,\left[\frac{\sqrt{2} \mu_{P}^{-}(x) \mu_{Q}^{-}(x)}{\sqrt{\left(\mu_{P}^{-}(x)\right)^{2}+\left(\mu_{Q}^{-}(x)\right)^{2}}}, \frac{\sqrt{2} \mu_{P}^{+}(x) \mu_{Q}^{+}(x)}{\sqrt{\left(\mu_{P}^{+}(x)\right)^{2}+\left(\mu_{Q}^{+}(x)\right)^{2}}}\right],\right.\right. \\
& \left.\left.\left[\frac{\sqrt{2} \nu_{P}^{-}(x) \nu_{Q}^{-}(x)}{\sqrt{\left(\nu_{P}^{-}(x)\right)^{2}+\left(\nu_{Q}^{-}(x)\right)^{2}}}, \frac{\sqrt{2} \nu_{P}^{+}(x) \nu_{Q}^{+}(x)}{\sqrt{\left(\nu_{P}^{+}(x)\right)^{2}+\left(\nu_{Q}^{+}(x)\right)^{2}}}\right]\right\rangle \mid x \in X\right\} ;
\end{aligned}
$$

5. $P \Psi Q=$

$$
\begin{aligned}
& \left\{\left\langlex,\left[\sqrt{\frac{\left(\mu_{P}^{-}(x)\right)^{2}+\left(\mu_{Q}^{-}(x)\right)^{2}}{2\left(\left(\mu_{P}^{-}(x)\right)^{2}+\left(\mu_{Q}^{-}(x)\right)^{2}+1\right)}},\right.\right.\right. \\
& \left.\sqrt{\frac{\left(\mu_{P}^{+}(x)\right)^{2}+\left(\mu_{Q}^{+}(x)\right)^{2}}{2\left(\left(\mu_{P}^{+}(x)\right)^{2}+\left(\mu_{Q}^{+}(x)\right)^{2}+1\right)}}\right],\left[\sqrt{\frac{\left(\nu_{P}^{-}(x)\right)^{2}+\left(\nu_{Q}^{-}(x)\right)^{2}}{2\left(\left(\nu_{P}^{-}(x)\right)^{2}+\left(\nu_{Q}^{-}(x)\right)^{2}+1\right)}}\right. \\
& \sqrt{\left.\left.\left.\frac{\left(\nu_{P}^{+}(x)\right)^{2}+\left(\nu_{Q}^{+}(x)\right)^{2}}{2\left(\left(\nu_{P}^{+}(x)\right)^{2}+\left(\nu_{Q}^{+}(x)\right)^{2}+1\right)}\right]\right\rangle \mid x \in X\right\} .}
\end{aligned}
$$

Example 3. Let $P$ and $Q$ be two IVPFSs over $X$ such that:

$$
\begin{aligned}
P=\{ & <x_{1},[0.2,0.3],[0.4,0.6]>, \\
& <x_{2},[0.2,0.4],[0.5,0.8]>, \\
& <x_{3},[0.4,0.5],[0.6,0.7]>, \\
& \left.<x_{4},[0.5,0.6],[0.7,0.8]>\right\},
\end{aligned}
$$

and:

$$
\begin{aligned}
Q=\{ & <x_{1},[0.2,0.4],[0.4,0.9]>, \\
& <x_{2},[0.3,0.4],[0.5,0.8]>, \\
& <x_{3},[0.5,0.6],[0.6,0.7]>, \\
& \left.<x_{4},[0.5,0.7],[0.6,0.7]>\right\} .
\end{aligned}
$$

Then, we can easily compute $P \boldsymbol{Q}, P \rightarrow Q, P \$ Q$, $P \& Q$ and $P \&$ by the above definitions shown in Table 1.

Lemma 1. For any three numbers $x, y, z \in[0,1]$, then:

$$
z^{2}\left(2-x^{2}-y^{2}-z^{2}\right)+x^{2} y^{2} \geq 0 .
$$

\section{Proof}

(1) If $x y \geq z^{2}$, then:

$$
\begin{aligned}
& z^{2}\left(2-x^{2}-y^{2}-z^{2}\right)+x^{2} y^{2}=z^{2}\left(2-x^{2}-y^{2}\right) \\
& -z^{4}+x^{2} y^{2} \geq-w^{4}+x^{2} y^{2} \geq 0 .
\end{aligned}
$$

(2) If $x y \leq z^{2}$, then: 
Table 1. The results of $\boldsymbol{\phi}, \rightarrow, \$, \mathbf{s}, \boldsymbol{\Psi}$ operations on $P$ and $Q$.

\begin{tabular}{cl}
\hline Operations & \multicolumn{1}{c}{ Results } \\
\hline$P \wedge Q$ & $\left\{<x_{1},[0.2,0.3536],[0.4,0.7649]>,<x_{2},[0.2550,0.4],[0.5,0.8]>\right.$, \\
& $\left.<x_{3},[0.4528,0.5523],[0.6,0.7]>,<x_{4},[0.5,0.6519],[0.6519,0.7517]>\right\}$ \\
$P \rightarrow Q \quad\left\{x_{1},[0.4,0.6],[0.2,0.3]>,<x_{2},[0.5,0.8],[0.2,0.4]>\right.$, \\
& $\left.<x_{3},[0.6,0.7],[0.4,0.5]>,<x_{4},[0.7,0.8],[0.5,0.6]>\right\}$ \\
& $\left\{<x_{1},[0.2,0.3464],[0.4,0.7348]>,<x_{2},[0.2449,0.4],[0.5,0.8]>\right.$, \\
& $\left.<x_{3},[0.4472,0.5477],[0.6,0.7]>,<x_{4},[0.5,0.6481],[0.6481,0.7483]>\right\}$ \\
& $\left\{<x_{1},[0.2,0.3394],[0.4,0.7060]>,<x_{2},[0.2353,0.4],[0.5,0.8]>\right.$, \\
& $\left.<x_{3},[0.4417,0.5432],[0.6,0.7]>,<x_{4},[0.5,0.6443],[0.6443,0.7450]>\right\}$ \\
& $\left\{<x_{1},[0.1925,0.3162],[0.3482,0.5192]>,<x_{2},[0.2398,0.3482],[0.4082,0.5298]>\right.$, \\
& $\left.<x_{3},[0.3813,0.4352],[0.4575,0.4975]>,<x_{4},[0.4082,0.4793],[0.4793,0.5150]>\right\}$ \\
&
\end{tabular}

$$
\begin{aligned}
z^{2}(2 & \left.-x^{2}-y^{2}-z^{2}\right)+x^{2} y^{2} \geq x y\left(2-x^{2}-y^{2}-z^{2}\right) \\
& +x^{2} y^{2}=x y\left(1-x^{2}-y^{2}+1-z^{2}+x y\right) \\
& \geq x y\left(1-x^{2}-y^{2}+x y\right) \\
= & \left\{\begin{array}{l}
x y\left(1-x^{2}+y(x-y)\right) \geq 0, \quad \text { iff } x \geq y \\
x y\left(1-y^{2}+x(y-x)\right) \geq 0, \quad \text { iff } x \leq y
\end{array}\right.
\end{aligned}
$$

Combining (1) with (2), we can have $z^{2}\left(2-x^{2}-y^{2}-\right.$ $\left.z^{2}\right)+x^{2} y^{2} \geq 0$.

The proof is completed.

Lemma 2. For any two numbers $x, y \in[0,1]$ :

$0 \leq x+y-2 x y \leq 1$.

Theorem 1. Let $X$ be a nonempty set. For IVPFSs $P, Q$, and $R$ in $X$ :
(1) $(P \oplus Q) \boldsymbol{\oplus} R \subseteq(P \boldsymbol{\phi} R) \oplus(Q \boldsymbol{\leftrightarrow} R)$;
(2) $(P \otimes Q) \boldsymbol{A} R \supseteq(P \boldsymbol{\phi} R) \otimes Q \boldsymbol{A} R)$;
(3) $(P \oplus Q) \rightarrow R \subseteq(P \rightarrow R) \oplus(Q \rightarrow R)$;
(4) $(P \otimes Q) \rightarrow R \supseteq(P \rightarrow R) \otimes Q \rightarrow R)$;
(5) $(P \oplus Q) \$ R \subseteq(P \$ R) \oplus(Q \$ R)$;
(6) $(P \otimes Q) \$ R \supseteq(P \$ R) \otimes Q \$ R)$;

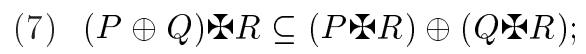
(8) $(P \otimes Q) \mathbb{\Psi} R \supseteq(P \mathbb{\Psi} R) \otimes Q \mathbb{\Psi} R)$.

Proof. We only prove (1) and (3) in detail. Statements (2), (4)-(8) can be proved in a similar way.

1. Let $P, Q$, and $R$ be three given IVPFSs, and then $(P \oplus Q) \boldsymbol{\phi} R$ and $(P \boldsymbol{\phi} R) \oplus(Q \boldsymbol{\oplus} R)$ are calculated as shown in Box I.
Let $f(x)$ be calculated as shown in Box II. According to Lemma 1 , we can have $f(x) \geq 0$.

Furthermore, we can have the relation shown in Box III. Let $g(x)$ be calculated as shown in Box IV. According to Lemma 1 , we can have $g(x) \geq 0$. Furthermore, we can have:

$$
\begin{aligned}
& \sqrt{\frac{\left(\nu_{P}^{-}(x)\right)^{2}\left(\nu_{Q}^{-}(x)\right)^{2}+\left(\nu_{R}^{-}(x)\right)^{2}}{2}} \\
& \geq \sqrt{\frac{\left(\nu_{P}^{-}(x)\right)^{2}+\left(\nu_{R}^{-}(x)\right)^{2}}{2} * \frac{\left(\nu_{Q}^{-}(x)\right)^{2}+\left(\nu_{R}^{-}(x)\right)^{2}}{2}} .
\end{aligned}
$$

Similarly we can have the relation shown in Box V. According to Eq. (5), we can have $(P \oplus Q) \boldsymbol{A} R \subseteq$ $(P \boldsymbol{A} R) \oplus(Q \boldsymbol{A} R)$.

3. Let $P, Q$, and $R$ be three given IVPFSs, then $(P \oplus$ $Q) \rightarrow R$ and $(P \rightarrow R) \oplus(Q \rightarrow R)$ be calculated as shown in Box VI.

Let $f(x)$ be calculated as shown in Box VII, then we can discuss the four cases in the following:

Case 1: If $\nu_{P}^{-}(x) \geq \nu_{Q}^{-}(x) \geq \mu_{R}^{-}(x)$ or $\nu_{Q}^{-}(x) \geq$ $\nu_{P}^{-}(x) \geq \mu_{R}^{-}(x)$, then:

Case 1.1: If $\nu_{P}^{-}(x) \nu_{Q}^{-}(x) \geq \mu_{R}^{-}(x)$, then:

$$
\begin{aligned}
f(x) & =\left(\nu_{P}^{-}(x)\right)^{2}\left(\nu_{Q}^{-}(x)\right)^{2}-\left(\left(\nu_{P}^{-}(x)\right)^{2}\right. \\
& \left.+\left(\nu_{Q}^{-}(x)\right)^{2}-\left(\nu_{P}^{-}(x)\right)^{2}\left(\nu_{Q}^{-}(x)\right)^{2}\right) \\
= & 2\left(\nu_{P}^{-}(x)\right)^{2}\left(\nu_{Q}^{-}(x)\right)^{2}-\left(\nu_{P}^{-}(x)\right)^{2} \\
& -\left(\nu_{Q}^{-}(x)\right)^{2} \leq 0 \quad(\text { Lemma 2). }
\end{aligned}
$$




$$
\begin{aligned}
& (P \oplus Q) \boldsymbol{\Lambda} R=\left\{\left\langlex,\left[\sqrt{\frac{\left(\mu_{P}^{-}(x)\right)^{2}+\left(\mu_{Q}^{-}(x)\right)^{2}-\left(\mu_{P}^{-}(x)\right)^{2}\left(\mu_{Q}^{-}(x)\right)^{2}+\left(\mu_{R}^{-}(x)\right)^{2}}{2}},\right.\right.\right. \\
& \left.\sqrt{\frac{\left(\mu_{P}^{+}(x)\right)^{2}+\left(\mu_{Q}^{+}(x)\right)^{2}-\left(\mu_{P}^{+}(x)\right)^{2}\left(\mu_{Q}^{+}(x)\right)^{2}+\left(\mu_{R}^{+}(x)\right)^{2}}{2}}\right] \\
& \left.\left.\left[\sqrt{\frac{\left(\nu_{P}^{-}(x)\right)^{2}\left(\nu_{Q}^{-}(x)\right)^{2}+\left(\nu_{R}^{-}(x)\right)^{2}}{2}}, \sqrt{\frac{\left(\nu_{P}^{+}(x)\right)^{2}\left(\nu_{Q}^{+}(x)\right)^{2}+\left(\nu_{R}^{+}(x)\right)^{2}}{2}}\right]\right\rangle x \in X\right\},
\end{aligned}
$$

$(P \wedge) \oplus(Q \mathbf{\wedge})$

$$
\begin{aligned}
&=\left\{\left\langlex,\left[\sqrt{\frac{\left(\mu_{P}^{-}(x)\right)^{2}+\left(\mu_{R}^{-}(x)\right)^{2}}{2}+\frac{\left(\mu_{Q}^{-}(x)\right)^{2}+\left(\mu_{R}^{-}(x)\right)^{2}}{2}-\frac{\left(\mu_{P}^{-}(x)\right)^{2}+\left(\mu_{R}^{-}(x)\right)^{2}}{2} * \frac{\left(\mu_{Q}^{-}(x)\right)^{2}+\left(\mu_{R}^{-}(x)\right)^{2}}{2}}\right.\right.\right. \\
&\left.\sqrt{\frac{\left(\mu_{P}^{+}(x)\right)^{2}+\left(\mu_{R}^{+}(x)\right)^{2}}{2}+\frac{\left(\mu_{Q}^{+}(x)\right)^{2}+\left(\mu_{R}^{+}(x)\right)^{2}}{2}-\frac{\left(\mu_{P}^{+}(x)\right)^{2}+\left(\mu_{R}^{+}(x)\right)^{2}}{2} * \frac{\left(\mu_{Q}^{+}(x)\right)^{2}+\left(\mu_{R}^{+}(x)\right)^{2}}{2}}\right], \\
& {\left.\left.\left[\sqrt{\frac{\left(\nu_{P}^{-}(x)\right)^{2}+\left(\nu_{R}^{-}(x)\right)^{2}}{2} * \frac{\left(\nu_{Q}^{-}(x)\right)^{2}+\left(\nu_{R}^{-}(x)\right)^{2}}{2}}, \sqrt{\frac{\left(\nu_{P}^{+}(x)\right)^{2}+\left(\nu_{R}^{+}(x)\right)^{2}}{2} * \frac{\left(\nu_{Q}^{+}(x)\right)^{2}+\left(\nu_{R}^{+}(x)\right)^{2}}{2}}\right]\right\rangle x \in X\right\} . }
\end{aligned}
$$

\section{Box I}

$$
\begin{aligned}
f(x)= & \left(\sqrt{\frac{\left(\mu_{P}^{-}(x)\right)^{2}+\left(\mu_{R}^{-}(x)\right)^{2}}{2}+\frac{\left(\mu_{Q}^{-}(x)\right)^{2}+\left(\mu_{R}^{-}(x)\right)^{2}}{2}-\frac{\left(\mu_{P}^{-}(x)\right)^{2}+\left(\mu_{R}^{-}(x)\right)^{2}}{2} * \frac{\left(\mu_{Q}^{-}(x)\right)^{2}+\left(\mu_{R}^{-}(x)\right)^{2}}{2}}\right)^{2} \\
& -\left(\sqrt{\frac{\left(\mu_{P}^{-}(x)\right)^{2}+\left(\mu_{Q}^{-}(x)\right)^{2}-\left(\mu_{P}^{-}(x)\right)^{2}\left(\mu_{Q}^{-}(x)\right)^{2}+\left(\mu_{R}^{-}(x)\right)^{2}}{2}}\right)^{2} \\
= & \frac{\left(\mu_{P}^{-}(x)\right)^{2}\left(\mu_{Q}^{-}(x)\right)^{2}+\left(\mu_{R}^{-}(x)\right)^{2}\left(2-\left(\mu_{P}^{-}(x)\right)^{2}-\left(\mu_{Q}^{-}(x)\right)^{2}-\left(\mu_{R}^{-}(x)\right)^{2}\right)}{2} .
\end{aligned}
$$

Box II

$$
\begin{aligned}
& \sqrt{\frac{\left(\mu_{P}^{-}(x)\right)^{2}+\left(\mu_{R}^{-}(x)\right)^{2}}{2}+\frac{\left(\mu_{Q}^{-}(x)\right)^{2}+\left(\mu_{R}^{-}(x)\right)^{2}}{2}-\frac{\left(\mu_{P}^{-}(x)\right)^{2}+\left(\mu_{R}^{-}(x)\right)^{2}}{2} * \frac{\left(\mu_{Q}^{-}(x)\right)^{2}+\left(\mu_{R}^{-}(x)\right)^{2}}{2}} \\
& \geq \sqrt{\frac{\left(\mu_{P}^{-}(x)\right)^{2}+\left(\mu_{Q}^{-}(x)\right)^{2}-\left(\mu_{P}^{-}(x)\right)^{2}\left(\mu_{Q}^{-}(x)\right)^{2}+\left(\mu_{R}^{-}(x)\right)^{2}}{2}}
\end{aligned}
$$




$$
\begin{aligned}
g(x) & =\left(\sqrt{\frac{\left(\nu_{P}^{-}(x)\right)^{2}\left(\nu_{Q}^{-}(x)\right)^{2}+\left(\nu_{R}^{-}(x)\right)^{2}}{2}}\right)^{2}-\left(\sqrt{\frac{\left(\nu_{P}^{-}(x)\right)^{2}+\left(\nu_{R}^{-}(x)\right)^{2}}{2} * \frac{\left(\nu_{Q}^{-}(x)\right)^{2}+\left(\nu_{R}^{-}(x)\right)^{2}}{2}}\right)^{2} \\
& =\frac{\left(\nu_{P}^{-}(x)\right)^{2}\left(\nu_{Q}^{-}(x)\right)^{2}+\left(\nu_{R}^{-}(x)\right)^{2}\left(2-\left(\nu_{P}^{-}(x)\right)^{2}-\left(\nu_{Q}^{-}(x)\right)^{2}-\left(\nu_{R}^{-}(x)\right)^{2}\right)}{2} .
\end{aligned}
$$

\section{Box IV}

$$
\begin{aligned}
& \sqrt{\frac{\left(\mu_{P}^{+}(x)\right)^{2}+\left(\mu_{R}^{+}(x)\right)^{2}}{2}+\frac{\left(\mu_{Q}^{+}(x)\right)^{2}+\left(\mu_{R}^{+}(x)\right)^{2}}{2}-\frac{\left(\mu_{P}^{+}(x)\right)^{2}+\left(\mu_{R}^{+}(x)\right)^{2}}{2} * \frac{\left(\mu_{Q}^{+}(x)\right)^{2}+\left(\mu_{R}^{+}(x)\right)^{2}}{2}} \\
& \quad \geq \sqrt{\frac{\left(\mu_{P}^{+}(x)\right)^{2}+\left(\mu_{Q}^{+}(x)\right)^{2}-\left(\mu_{P}^{+}(x)\right)^{2}\left(\mu_{Q}^{+}(x)\right)^{2}+\left(\mu_{R}^{+}(x)\right)^{2}}{2}}, \sqrt{\frac{\left(\nu_{P}^{+}(x)\right)^{2}\left(\nu_{Q}^{+}(x)\right)^{2}+\left(\nu_{R}^{+}(x)\right)^{2}}{2}} \\
& \quad \geq \sqrt{\frac{\left(\nu_{P}^{+}(x)\right)^{2}+\left(\nu_{R}^{+}(x)\right)^{2}}{2} * \frac{\left(\nu_{Q}^{+}(x)\right)^{2}+\left(\nu_{R}^{+}(x)\right)^{2}}{2}}
\end{aligned}
$$

\section{Box V}

$$
\begin{aligned}
& (P \oplus Q) \rightarrow R=\left\{\left\langlex,\left[\max \left\{\nu_{P}^{-}(x) \nu_{Q}^{-}(x), \mu_{R}^{-}(x)\right\}, \max \left\{\nu_{P}^{+}(x) \nu_{Q}^{+}(x), \mu_{R}^{+}(x)\right\}\right],\right.\right. \\
& {\left[\min \left\{\sqrt{\left(\mu_{P}^{-}(x)\right)^{2}+\left(\mu_{Q}^{-}(x)\right)^{2}-\left(\mu_{P}^{-}(x)\right)^{2}\left(\mu_{Q}^{-}(x)\right)^{2}}, \nu_{R}^{-}(x)\right\},\right.} \\
& \left.\left.\left.\min \left\{\sqrt{\left(\mu_{P}^{+}(x)\right)^{2}+\left(\mu_{Q}^{+}(x)\right)^{2}-\left(\mu_{P}^{+}(x)\right)^{2}\left(\mu_{Q}^{+}(x)\right)^{2}}, \nu_{R}^{+}(x)\right\}\right]\right\rangle \mid x \in X\right\}, \\
& (P \rightarrow R) \oplus(Q \rightarrow R)=\left\{\left\langlex,\left[\begin{array}{r}
\begin{array}{r}
\max \left\{\left(\nu_{P}^{-}(x)\right)^{2},\left(\mu_{R}^{-}(x)\right)^{2}\right\}+\max \left\{\left(\nu_{Q}^{-}(x)\right)^{2},\left(\mu_{R}^{-}(x)\right)^{2}\right\} \\
-\max \left\{\left(\nu_{P}^{-}(x)\right)^{2},\left(\mu_{R}^{-}(x)\right)^{2}\right\} * \max \left\{\left(\nu_{Q}^{-}(x)\right)^{2},\left(\mu_{R}^{-}(x)\right)^{2}\right\}
\end{array}
\end{array}\right.\right.\right. \\
& \sqrt{\left.\frac{\max \left\{\left(\nu_{P}^{+}(x)\right)^{2},\left(\mu_{R}^{+}(x)\right)^{2}\right\}+\max \left\{\left(\nu_{Q}^{+}(x)\right)^{2},\left(\mu_{R}^{+}(x)\right)^{2}\right\}-\max \left\{\left(\nu_{P}^{+}(x)\right)^{2},\left(\mu_{R}^{+}(x)\right)^{2}\right\}}{* \max \left\{\left(\nu_{Q}^{+}(x)\right)^{2},\left(\mu_{R}^{+}(x)\right)^{2}\right\}}\right],} \\
& \left.\left.\left[\min \left\{\mu_{P}^{-}(x), \nu_{R}^{-}(x)\right\} * \min \left\{\mu_{Q}^{-}(x), \nu_{R}^{-}(x)\right\}, \min \left\{\mu_{P}^{+}(x), \nu_{R}^{+}(x)\right\} * \min \left\{\mu_{Q}^{+}(x), \nu_{R}^{+}(x)\right\}\right]\right\rangle \mid x \in X\right\} .
\end{aligned}
$$

\section{Box VI}

$$
\begin{aligned}
& f(x)=\left(\max \left\{\nu_{P}^{-}(x) \nu_{Q}^{-}(x), \mu_{R}^{-}(x)\right\}\right)^{2}-\left(\sqrt[\begin{array}{c}
\max \left\{\left(\nu_{P}^{-}(x)\right)^{2},\left(\mu_{R}^{-}(x)\right)^{2}\right\}+\max \left\{\left(\nu_{Q}^{-}(x)\right)^{2},\left(\mu_{R}^{-}(x)\right)^{2}\right\} \\
-\max \left\{\left(\nu_{P}^{-}(x)\right)^{2},\left(\mu_{R}^{-}(x)\right)^{2}\right\} * \max \left\{\left(\nu_{Q}^{-}(x)\right)^{2},\left(\mu_{R}^{-}(x)\right)^{2}\right\}
\end{array}]{{ }^{2}}\right. \\
& =\max \left\{\nu_{P}^{-}(x) \nu_{Q}^{-}(x), \mu_{R}^{-}(x)\right\}-\left(\max \left\{\left(\nu_{P}^{-}(x)\right)^{2},\left(\mu_{R}^{-}(x)\right)^{2}\right\}+\max \left\{\left(\nu_{Q}^{-}(x)\right)^{2},\left(\mu_{R}^{-}(x)\right)^{2}\right\}\right. \\
& \left.-\max \left\{\left(\nu_{P}^{-}(x)\right)^{2},\left(\mu_{R}^{-}(x)\right)^{2}\right\} * \max \left\{\left(\nu_{Q}^{-}(x)\right)^{2},\left(\mu_{R}^{-}(x)\right)^{2}\right\}\right) .
\end{aligned}
$$


Case 1.2: If $\nu_{P}^{-}(x) \nu_{Q}^{-}(x) \leq \mu_{R}^{-}(x)$, then:

$$
\begin{aligned}
f(x)= & \left(\mu_{R}^{-}(x)\right)^{2}-\left(\left(\nu_{P}^{-}(x)\right)^{2}+\left(\nu_{Q}^{-}(x)\right)^{2}\right. \\
& \left.-\left(\nu_{P}^{-}(x)\right)^{2}\left(\nu_{Q}^{-}(x)\right)^{2}\right) \\
= & \left(\left(\mu_{R}^{-}(x)\right)^{2}-\left(\nu_{P}^{-}(x)\right)^{2}\right) \\
& +\left(\nu_{Q}^{-}(x)\right)^{2}\left(\left(\nu_{P}^{-}(x)\right)^{2}-1\right) \\
& \leq 0\left(\left(\mu_{R}^{-}(x)\right)^{2}-\left(\nu_{P}^{-}(x)\right)^{2}\right. \\
& \left.\leq 0,\left(\nu_{P}^{-}(x)\right)^{2}-1 \leq 0\right) .
\end{aligned}
$$

Case 2: If $\nu_{P}^{-}(x) \geq \mu_{R}^{-}(x) \geq \nu_{Q}^{-}(x)$, then:

Case 2.1: If $\nu_{P}^{-}(x) \nu_{Q}^{-}(x) \geq \mu_{R}^{-}(x)$, then:

$$
\begin{aligned}
f(x)= & \left(\nu_{P}^{-}(x)\right)^{2}\left(\nu_{Q}^{-}(x)\right)^{2}-\left(\left(\nu_{P}^{-}(x)\right)^{2}\right. \\
& \left.+\left(\mu_{R}^{-}(x)\right)^{2}-\left(\nu_{P}^{-}(x)\right)^{2}\left(\mu_{R}^{-}(x)\right)^{2}\right) \\
= & \left(\nu_{P}^{-}(x)\right)^{2}\left(\left(\nu_{Q}^{-}(x)\right)^{2}-1\right) \\
& +\left(\mu_{R}^{-}(x)\right)^{2}\left(\left(\nu_{P}^{-}(x)\right)^{2}-1\right) \leq 0 .
\end{aligned}
$$

Case 2.2: If $\nu_{P}^{-}(x) \nu_{Q}^{-}(x) \leq \mu_{R}^{-}(x)$, then:

$$
\begin{aligned}
f(x)= & \left(\mu_{R}^{-}(x)\right)^{2}-\left(\left(\nu_{P}^{-}(x)\right)^{2}+\left(\mu_{R}^{-}(x)\right)^{2}\right. \\
& \left.-\left(\nu_{P}^{-}(x)\right)^{2}\left(\mu_{R}^{-}(x)\right)^{2}\right) \\
= & \left(\nu_{P}^{-}(x)\right)^{2}\left(\left(\mu_{R}^{-}(x)\right)^{2}-1\right) \leq 0 .
\end{aligned}
$$

Case 3: If $\nu_{Q}^{-}(x) \geq \mu_{R}^{-}(x) \geq \nu_{P}^{-}(x)$, then:

Case 3.1: If $\nu_{P}^{-}(x) \nu_{Q}^{-}(x) \geq \mu_{R}^{-}(x)$, then:

$$
\begin{aligned}
f(x)= & \left(\nu_{P}^{-}(x)\right)^{2}\left(\nu_{Q}^{-}(x)\right)^{2}-\left(\left(\mu_{R}^{-}(x)\right)^{2}\right. \\
& \left.+\left(\nu_{Q}^{-}(x)\right)^{2}-\left(\mu_{R}^{-}(x)\right)^{2}\left(\nu_{Q}^{-}(x)\right)^{2}\right) \\
= & \left(\nu_{Q}^{-}(x)\right)^{2}\left(\left(\nu_{P}^{-}(x)\right)^{2}-1\right) \\
& +\left(\mu_{R}^{-}(x)\right)^{2}\left(\left(\nu_{Q}^{-}(x)\right)^{2}-1\right) \leq 0 .
\end{aligned}
$$

Case 3.2: If $\nu_{P}^{-}(x) \nu_{Q}^{-}(x) \leq \mu_{R}^{-}(x)$, then:

$$
\begin{aligned}
f(x)= & \left(\mu_{R}^{-}(x)\right)^{2}-\left(\left(\mu_{R}^{-}(x)\right)^{2}+\left(\nu_{Q}^{-}(x)\right)^{2}\right. \\
& \left.-\left(\mu_{R}^{-}(x)\right)^{2}\left(\nu_{Q}^{-}(x)\right)^{2}\right) \\
= & \left(\nu_{Q}^{-}(x)\right)^{2}\left(\left(\mu_{R}^{-}(x)\right)^{2}-1\right) \leq 0 .
\end{aligned}
$$

Case 4: If $\mu_{R}^{-}(x) \geq \nu_{P}^{-}(x) \geq \nu_{Q}^{-}(x)$ or $\mu_{R}^{-}(x) \geq$ $\nu_{Q}^{-}(x) \geq \nu_{P}^{-}(x)$, then:
Case 4.1: If $\nu_{P}^{-}(x) \nu_{Q}^{-}(x) \geq \mu_{R}^{-}(x)$, then:

$$
\begin{aligned}
& f(x)=\left(\nu_{P}^{-}(x)\right)^{2}\left(\nu_{Q}^{-}(x)\right)^{2}-\left(\left(\mu_{R}^{-}(x)\right)^{2}\right. \\
&\left.+\left(\mu_{R}^{-}(x)\right)^{2}-\left(\mu_{R}^{-}(x)\right)^{2}\left(\mu_{R}^{-}(x)\right)^{2}\right) \\
&=\left(\nu_{P}^{-}(x)\right)^{2}\left(\nu_{Q}^{-}(x)\right)^{2}+\left(\mu_{R}^{-}(x)\right)^{2}\left(\mu_{R}^{-}(x)\right)^{2} \\
& \quad-2\left(\mu_{R}^{-}(x)\right)^{2} \leq\left(\mu_{R}^{-}(x)\right)^{2}\left(\mu_{R}^{-}(x)\right)^{2} \\
& \quad+\left(\mu_{R}^{-}(x)\right)^{2}\left(\mu_{R}^{-}(x)\right)^{2}-2\left(\mu_{R}^{-}(x)\right)^{2}\left(\nu_{P}^{-}(x)\right. \\
&\left.\leq \mu_{R}^{-}(x), \nu_{Q}^{-}(x) \leq \mu_{R}^{-}(x)\right) \\
&=2\left(\mu_{R}^{-}(x)\right)^{2}\left(\left(\mu_{R}^{-}(x)\right)^{2}-1\right) \leq 0 .
\end{aligned}
$$

Case 4.2: If $\nu_{P}^{-}(x) \nu_{Q}^{-}(x) \leq \mu_{R}^{-}(x)$, then:

$$
\begin{aligned}
f(x)= & \left(\mu_{R}^{-}(x)\right)^{2}-\left(\left(\mu_{R}^{-}(x)\right)^{2}+\left(\mu_{R}^{-}(x)\right)^{2}\right. \\
& \left.-\left(\mu_{R}^{-}(x)\right)^{2}\left(\mu_{R}^{-}(x)\right)^{2}\right) \\
= & \left(\mu_{R}^{-}(x)\right)^{2}\left(\left(\mu_{R}^{-}(x)\right)^{2}-1\right) \leq 0 .
\end{aligned}
$$

From above four cases, we can have $f(x) \leq 0$, i.e.:

$$
\begin{aligned}
& \max \left\{\left(\nu_{P}^{-}(x)\right)^{2},\left(\mu_{R}^{-}(x)\right)^{2}\right\} \\
& +\max \left\{\left(\nu_{Q}^{-}(x)\right)^{2},\left(\mu_{R}^{-}(x)\right)^{2}\right\} \\
& -\max \left\{\left(\nu_{P}^{-}(x)\right)^{2},\left(\mu_{R}^{-}(x)\right)^{2}\right\} \\
& \sqrt{ } * \max \left\{\left(\nu_{Q}^{-}(x)\right)^{2},\left(\mu_{R}^{-}(x)\right)^{2}\right\} \\
& \geq \max \left\{\nu_{P}^{-}(x) \nu_{Q}^{-}(x), \mu_{R}^{-}(x)\right\} .
\end{aligned}
$$

Similarly:

$$
\begin{array}{r}
\max \left\{\left(\nu_{P}^{+}(x)\right)^{2},\left(\mu_{R}^{+}(x)\right)^{2}\right\} \\
+\max \left\{\left(\nu_{Q}^{+}(x)\right)^{2},\left(\mu_{R}^{+}(x)\right)^{2}\right\} \\
-\max \left\{\left(\nu_{P}^{+}(x)\right)^{2},\left(\mu_{R}^{+}(x)\right)^{2}\right\} \\
\quad * \max \left\{\left(\nu_{Q}^{+}(x)\right)^{2},\left(\mu_{R}^{+}(x)\right)^{2}\right\} \\
\geq \max \left\{\nu_{P}^{+}(x) \nu_{Q}^{+}(x), \mu_{R}^{+}(x)\right\} .
\end{array}
$$

Let:

$$
\begin{aligned}
g(x)= & \min \left\{\left(\mu_{P}^{-}(x)\right)^{2}+\left(\mu_{Q}^{-}(x)\right)^{2}\right. \\
& \left.-\left(\mu_{P}^{-}(x)\right)^{2}\left(\mu_{Q}^{-}(x)\right)^{2},\left(\nu_{R}^{-}(x)\right)^{2}\right\} \\
& -\min \left\{\left(\mu_{P}^{-}(x)\right)^{2},\left(\nu_{R}^{-}(x)\right)^{2}\right\} \\
& \min \left\{\left(\mu_{Q}^{-}(x)\right)^{2},\left(\nu_{R}^{-}(x)\right)^{2}\right\},
\end{aligned}
$$

then: 
Case 1: If $\mu_{P}^{-}(x) \geq \mu_{Q}^{-}(x) \geq \nu_{R}^{-}(x)$ or $\mu_{Q}^{-}(x) \geq$ $\mu_{P}^{-}(x) \geq \nu_{R}^{-}(x)$, then:

$$
\begin{aligned}
\left(\mu_{P}^{-}(x)\right)^{2} & +\left(\mu_{Q}^{-}(x)\right)^{2}-\left(\mu_{P}^{-}(x)\right)^{2}\left(\mu_{Q}^{-}(x)\right)^{2} \\
& -\left(\nu_{R}^{-}(x)\right)^{2}=\left(\mu_{P}^{-}(x)\right)^{2}-\left(\nu_{R}^{-}(x)\right)^{2} \\
& +\left(\mu_{Q}^{-}(x)\right)^{2}\left(1-\left(\mu_{P}^{-}(x)\right)^{2}\right) \\
& \geq 0\left(\mu_{P}^{-}(x) \geq \nu_{R}^{-}(x), 1-\left(\mu_{P}^{-}(x)\right)^{2} \geq 0\right) .
\end{aligned}
$$

Hence:

$$
\begin{aligned}
g(x) & =\left(\nu_{R}^{-}(x)\right)^{2}-\left(\nu_{R}^{-}(x)\right)^{4} \\
& =\left(\nu_{R}^{-}(x)\right)^{2}\left(1-\left(\nu_{R}^{-}(x)\right)^{2}\right) \geq 0 .
\end{aligned}
$$

Case 2: If $\mu_{P}^{-}(x) \geq \nu_{R}^{-}(x) \geq \mu_{Q}^{-}(x)$, then:

$$
\begin{aligned}
g(x) & =\left(\nu_{R}^{-}(x)\right)^{2}-\left(\nu_{R}^{-}(x)\right)^{2}\left(\mu_{Q}^{-}(x)\right)^{2} \\
& =\left(\nu_{R}^{-}(x)\right)^{2}\left(1-\left(\mu_{Q}^{-}(x)\right)^{2}\right) \geq 0 .
\end{aligned}
$$

Case 3: If $\mu_{Q}^{-}(x) \geq \nu_{R}^{-}(x) \geq \mu_{P}^{-}(x)$, then:

$$
\begin{aligned}
\left(\mu_{P}^{-}(x)\right)^{2} & +\left(\mu_{Q}^{-}(x)\right)^{2}-\left(\mu_{P}^{-}(x)\right)^{2}\left(\mu_{Q}^{-}(x)\right)^{2} \\
& -\left(\nu_{R}^{-}(x)\right)^{2}=\left(\mu_{Q}^{-}(x)\right)^{2}-\left(\nu_{R}^{-}(x)\right)^{2} \\
& +\left(\mu_{P}^{-}(x)\right)^{2}\left(1-\left(\mu_{Q}^{-}(x)\right)^{2}\right) \\
& \geq 0\left(\mu_{Q}^{-}(x) \geq \nu_{R}^{-}(x), 1-\left(\mu_{Q}^{-}(x)\right)^{2} \geq 0\right) .
\end{aligned}
$$

Hence:

$$
\begin{aligned}
g(x) & =\left(\nu_{R}^{-}(x)\right)^{2}-\left(\nu_{R}^{-}(x)\right)^{2}\left(\mu_{P}^{-}(x)\right)^{2} \\
& =\left(\nu_{R}^{-}(x)\right)^{2}\left(1-\left(\mu_{P}^{-}(x)\right)^{2}\right) \geq 0 .
\end{aligned}
$$

Case 4: If $\nu_{R}^{-}(x) \geq \mu_{Q}^{-}(x) \geq \mu_{P}^{-}(x)$ or $\nu_{R}^{-}(x) \geq$ $\mu_{P}^{-}(x) \geq \mu_{Q}^{-}(x)$, then:

Case 4.1: If $\left(\mu_{P}^{-}(x)\right)^{2}+\left(\mu_{Q}^{-}(x)\right)^{2}-\left(\mu_{P}^{-}(x)\right)^{2}$ $\left(\mu_{Q}^{-}(x)\right)^{2} \leq\left(\nu_{R}^{-}(x)\right)^{2}$, then:

$$
\begin{aligned}
g(x)= & \left(\mu_{P}^{-}(x)\right)^{2}+\left(\mu_{Q}^{-}(x)\right)^{2}-\left(\mu_{P}^{-}(x)\right)^{2}\left(\mu_{Q}^{-}(x)\right)^{2} \\
& -\left(\mu_{P}^{-}(x)\right)^{2}\left(\mu_{Q}^{-}(x)\right)^{2}=\left(\mu_{P}^{-}(x)\right)^{2} \\
& +\left(\mu_{Q}^{-}(x)\right)^{2}-2\left(\mu_{P}^{-}(x)\right)^{2}\left(\mu_{Q}^{-}(x)\right)^{2} \geq 0 .
\end{aligned}
$$

(Lemma 2).

Case 4.2: If $\left(\mu_{P}^{-}(x)\right)^{2}+\left(\mu_{Q}^{-}(x)\right)^{2}-\left(\mu_{P}^{-}(x)\right)^{2}$ $\left(\mu_{Q}^{-}(x)\right)^{2} \geq\left(\nu_{R}^{-}(x)\right)^{2}$, then:

$$
\begin{aligned}
g(x)= & \left(\nu_{R}^{-}(x)\right)^{2}-\left(\mu_{P}^{-}(x)\right)^{2}\left(\mu_{Q}^{-}(x)\right)^{2} \\
& \geq 0\left(\nu_{R}^{-}(x)>\mu_{P}^{-}(x), \nu_{R}^{-}(x)>\mu_{Q}^{-}(x)\right) .
\end{aligned}
$$

From the above four cases, we can have $g(x) \geq 0$, i.e.: $\min$

$$
\begin{aligned}
& \left\{\sqrt{\left(\mu_{P}^{-}(x)\right)^{2}+\left(\mu_{Q}^{-}(x)\right)^{2}-\left(\mu_{P}^{-}(x)\right)^{2}\left(\mu_{Q}^{-}(x)\right)^{2}}, \nu_{R}^{-}(x)\right\} \\
& \geq \min \left\{\mu_{P}^{-}(x), \nu_{R}^{-}(x)\right\} * \min \left\{\mu_{Q}^{-}(x), \nu_{R}^{-}(x)\right\} .
\end{aligned}
$$

Similarly:

$\min$

$$
\begin{aligned}
& \left\{\sqrt{\left(\mu_{P}^{+}(x)\right)^{2}+\left(\mu_{Q}^{+}(x)\right)^{2}-\left(\mu_{P}^{+}(x)\right)^{2}\left(\mu_{Q}^{+}(x)\right)^{2}}, \nu_{R}^{+}(x)\right\} \\
& \geq \min \left\{\mu_{P}^{+}(x), \nu_{R}^{+}(x)\right\} * \min \left\{\mu_{Q}^{+}(x), \nu_{R}^{+}(x)\right\} .
\end{aligned}
$$

Hence, according to Eq. (5), we can have:

$$
(P \oplus Q) \rightarrow R \subseteq(P \rightarrow R) \oplus(Q \rightarrow R) .
$$

Theorem 2. Let $X$ be a nonempty set. For IVPFSs $P, Q$ and $R$ in $X$, then:

(1) $(P \oplus Q) \oplus R=(P \oplus R) \boldsymbol{\oplus}(Q \oplus R)$;

(2) $(P \wedge Q) \otimes R=(P \otimes R) \wedge(Q \otimes R)$;

(3) $(P \$ Q) \oplus R \subseteq(P \oplus R) \$(Q \oplus R)$;

(4) $(P \$ Q) \otimes R \supseteq(P \otimes R) \$(Q \otimes R)$;

(5) $(P \boldsymbol{Q} Q) \oplus R \supseteq(P \oplus R) \boldsymbol{中}(Q \oplus R)$;

(6) $(P \boldsymbol{Q} Q) \otimes R \subseteq(P \otimes R) \boldsymbol{2}(Q \otimes R)$;

(7) $(P \uplus Q) \oplus R \supseteq(P \oplus R) \oplus(Q \oplus R)$;

(8) $(P \otimes Q) \otimes R \subseteq(P \otimes R) \otimes(Q \otimes R)$.

Proof. We only prove (1) and (5) in detail, and Statements (2)-(4) and (6)-(8) can be proved in a similar way.

(1) Let $P, Q$ and $R$ be three given IVPFSs, then $(P \oplus Q) \oplus R$ and $(P \oplus R) \oplus(Q \oplus R)$ are calculated as shown in Box VIII.

(5) Let $P, Q$, and $R$ be three given IVPFSs, then $(P \boldsymbol{Q} Q) \oplus R$ and $(P \oplus R) \boldsymbol{Q}(Q \oplus R)$ are calculated as shown in Box IX.

Let $f(x)$ be calculated as shown in Box X; then, it is calculated as shown in Boxes XI and XII.

Hence, we can have the relations shown in Box XIII. Therefore, according to Eq. (5), we can have:

$(P \boldsymbol{\leftrightarrow} Q) \oplus R \subseteq(P \oplus R) \boldsymbol{H}(Q \oplus R)$.

Definition 10. Let us define the generalized operator over the IVPFS $P_{i}(i=1,2, \cdots, n)$ by: 


$$
\begin{aligned}
(P \boldsymbol{\phi} Q) \oplus R= & \left\{\left\langlex,\left[\sqrt{\frac{\left(\mu_{P}^{-}(x)\right)^{2}+\left(\mu_{Q}^{-}(x)\right)^{2}}{2}+\left(\mu_{R}^{-}(x)\right)^{2}-\frac{\left(\mu_{P}^{-}(x)\right)^{2}+\left(\mu_{Q}^{-}(x)\right)^{2}}{2}\left(\mu_{R}^{-}(x)\right)^{2},},\right.\right.\right. \\
& \left.\sqrt{\frac{\left(\mu_{P}^{+}(x)\right)^{2}+\left(\mu_{Q}^{+}(x)\right)^{2}}{2}+\left(\mu_{R}^{+}(x)\right)^{2}-\frac{\left(\mu_{P}^{+}(x)\right)^{2}+\left(\mu_{Q}^{+}(x)\right)^{2}}{2}\left(\mu_{R}^{+}(x)\right)^{2}}\right], \\
& {\left.\left.\left[\sqrt{\frac{\left(\nu_{P}^{-}(x)\right)^{2}+\left(\nu_{Q}^{-}(x)\right)^{2}}{2}} \nu_{R}^{-}(x), \sqrt{\frac{\left(\nu_{P}^{+}(x)\right)^{2}+\left(\nu_{Q}^{+}(x)\right)^{2}}{2}} \nu_{R}^{+}(x)\right]\right\rangle x \in X\right\}, }
\end{aligned}
$$

$(P \oplus R) \mathbf{\oplus}(Q \oplus R)$

$$
\begin{aligned}
=\left\{\left\langlex,\left[\sqrt{\frac{\left(\mu_{P}^{-}(x)\right)^{2}+\left(\mu_{R}^{-}(x)\right)^{2}-\left(\mu_{P}^{-}(x)\right)^{2}\left(\mu_{R}^{-}(x)\right)^{2}+\left(\mu_{Q}^{-}(x)\right)^{2}+\left(\mu_{R}^{-}(x)\right)^{2}-\left(\mu_{Q}^{-}(x)\right)^{2}\left(\mu_{R}^{-}(x)\right)^{2}}{2},}\right.\right.\right. & \left.\sqrt{\frac{\left(\mu_{P}^{+}(x)\right)^{2}+\left(\mu_{R}^{+}(x)\right)^{2}-\left(\mu_{P}^{+}(x)\right)^{2}\left(\mu_{R}^{+}(x)\right)^{2}+\left(\mu_{Q}^{+}(x)\right)^{2}+\left(\mu_{R}^{+}(x)\right)^{2}-\left(\mu_{Q}^{+}(x)\right)^{2}\left(\mu_{R}^{+}(x)\right)^{2}}{2}}\right], \\
= & \left.\left.\left\{\sqrt{\frac{\left(\nu_{P}^{-}(x)\right)^{2}\left(\nu_{Q}^{-}(x)\right)^{2}+\left(\nu_{R}^{-}(x)\right)^{2}\left(\nu_{Q}^{-}(x)\right)^{2}}{2}}, \sqrt{\frac{\left(\nu_{P}^{+}(x)\right)^{2}\left(\nu_{Q}^{+}(x)\right)^{2}+\left(\nu_{R}^{+}(x)\right)^{2}\left(\nu_{Q}^{+}(x)\right)^{2}}{2}}\right]\right\rangle x \in X\right\} \\
& \left.\sqrt{\frac{\left(\mu_{P}^{+}(x)\right)^{2}+\left(\mu_{Q}^{+}(x)\right)^{2}}{2}+\left(\mu_{R}^{+}(x)\right)^{2}-\frac{\left(\mu_{P}^{+}(x)\right)^{2}+\left(\mu_{Q}^{+}(x)\right)^{2}}{2}\left(\mu_{R}^{+}(x)\right)^{2}}\right], \\
& {\left[\sqrt{\frac{\left(\mu_{P}^{-}(x)\right)^{2}+\left(\mu_{Q}^{-}(x)\right)^{2}}{2}+\left(\mu_{R}^{-}(x)\right)^{2}-\frac{\left(\mu_{P}^{-}(x)\right)^{2}+\left(\mu_{Q}^{-}(x)\right)^{2}}{2}\left(\mu_{R}^{-}(x)\right)^{2},}\right.} \\
& {\left.\left.\left[\sqrt{\frac{\left(\nu_{Q}^{-}(x)\right)^{2}}{2}} \nu_{R}^{-}(x), \sqrt{\frac{\left(\nu_{P}^{+}(x)\right)^{2}+\left(\nu_{Q}^{+}(x)\right)^{2}}{2}} \nu_{R}^{+}(x)\right]\right\rangle x \in X\right\}=(P \oplus R) \boldsymbol{(}(Q \oplus R) . }
\end{aligned}
$$

\section{Box VIII}

$$
\begin{gathered}
{\underset{i=1}{n} P_{i}}_{i=1}^{n}\left\{x,\left[\sqrt{\frac{\sum_{i=1}^{n}\left(\mu_{P_{i}}^{-}(x)\right)^{2}}{n}}, \sqrt{\frac{\sum_{i=1}^{n}\left(\mu_{P_{i}}^{+}(x)\right)^{2}}{n}}\right],\right. \\
\left.\left.\left[\sqrt{\frac{\sum_{i=1}^{n}\left(\nu_{P_{i}}^{-}(x)\right)^{2}}{n}}, \sqrt{\frac{\sum_{i=1}^{n}\left(\nu_{P_{i}}^{+}(x)\right)^{2}}{n}}\right]\right\rangle x \in X\right\} .
\end{gathered}
$$

When $n=2$, it reduces to $\prod_{i=1}^{2} P_{i}=P_{1} P_{2}$ defined above.

Theorem 3. For every IVPFS $P_{i}(i=1,2, \cdots, n)$ and $Q$ :
(1) $\left(\prod_{i=1}^{n} P_{i}^{c}\right)^{c}=\overbrace{i=1}^{n} P_{i}$;

(2) $\mathfrak{i = 1}_{i=1}^{n} P_{i} \oplus Q=\prod_{i=1}^{n}\left(P_{i} \oplus Q\right)$;

(3) $\prod_{i=1}^{n} P_{i} \otimes Q=\prod_{i=1}^{n}\left(P_{i} \otimes Q\right)$;

(4) $\square(\overbrace{i=1}^{n} P_{i})=\prod_{i=1}^{n} \square P_{i}$;

(5) $\diamond\left(\stackrel{\boldsymbol{\$}}{i=1}_{i}^{n} P_{i}\right)=\prod_{i=1}^{n} \diamond P_{i}$.

Proof. It is trivial. 
$(P \boldsymbol{\phi} Q) \oplus R$

$$
\begin{aligned}
= & \left\{\left\langlex,\left[\sqrt{\frac{2\left(\mu_{P}^{-}(x)\right)^{2}\left(\mu_{Q}^{-}(x)\right)^{2}+\left(\mu_{P}^{-}(x)\right)^{2}\left(\mu_{R}^{-}(x)\right)^{2}+\left(\mu_{Q}^{-}(x)\right)^{2}\left(\mu_{R}^{-}(x)\right)^{2}-2\left(\mu_{P}^{-}(x)\right)^{2}\left(\mu_{Q}^{-}(x)\right)^{2}\left(\mu_{R}^{-}(x)\right)^{2}}{\left(\mu_{P}^{-}(x)\right)^{2}+\left(\mu_{Q}^{-}(x)\right)^{2}},}\right.\right.\right. \\
& \sqrt{\left.\frac{2\left(\mu_{P}^{+}(x)\right)^{2}\left(\mu_{Q}^{+}(x)\right)^{2}+\left(\mu_{P}^{+}(x)\right)^{2}\left(\mu_{R}^{+}(x)\right)^{2}+\left(\mu_{Q}^{+}(x)\right)^{2}\left(\mu_{R}^{+}(x)\right)^{2}-2\left(\mu_{P}^{+}(x)\right)^{2}\left(\mu_{Q}^{+}(x)\right)^{2}\left(\mu_{R}^{+}(x)\right)^{2}}{\left(\mu_{P}^{+}(x)\right)^{2}+\left(\mu_{Q}^{+}(x)\right)^{2}}\right],} \\
& {\left.\left.\left[\frac{\sqrt{2} \nu_{P}^{-}(x) \nu_{Q}^{-}(x) \nu_{R}^{-}(x)}{\sqrt{\left(\nu_{P}^{-}(x)\right)^{2}+\left(\nu_{Q}^{-}(x)\right)^{2}}}, \frac{\sqrt{2} \nu_{P}^{+}(x) \nu_{Q}^{+}(x) \nu_{R}^{+}(x)}{\sqrt{\left(\nu_{P}^{+}(x)\right)^{2}+\left(\nu_{Q}^{+}(x)\right)^{2}}}\right]\right\rangle x \in X\right\}, }
\end{aligned}
$$

$(P \oplus R) \boldsymbol{p}(Q \oplus R)$

$$
\begin{aligned}
= & \left\{\left\langlex,\left[\sqrt{\frac{2\left(\left(\mu_{P}^{-}(x)\right)^{2}+\left(\mu_{R}^{-}(x)\right)^{2}-\left(\mu_{P}^{-}(x)\right)^{2}\left(\mu_{R}^{-}(x)\right)^{2}\right)\left(\left(\mu_{Q}^{-}(x)\right)^{2}+\left(\mu_{R}^{-}(x)\right)^{2}-\left(\mu_{Q}^{-}(x)\right)^{2}\left(\mu_{R}^{-}(x)\right)^{2}\right)}{\left(\mu_{P}^{-}(x)\left(\mu_{Q}^{-}(x)\right)^{2}+2\left(\mu_{R}^{-}(x)\right)^{2}-\left(\mu_{P}^{-}(x)\right)^{2}\left(\mu_{R}^{-}(x)\right)^{2}-\left(\mu_{Q}^{-}(x)\right)^{2}\left(\mu_{R}^{-}(x)\right)^{2}\right.},}\right.\right.\right. \\
& \sqrt{\left.\frac{2\left(\left(\mu_{P}^{+}(x)\right)^{2}+\left(\mu_{R}^{+}(x)\right)^{2}-\left(\mu_{P}^{+}(x)\right)^{2}\left(\mu_{R}^{+}(x)\right)^{2}\right)\left(\left(\mu_{Q}^{+}(x)\right)^{2}+\left(\mu_{R}^{+}(x)\right)^{2}-\left(\mu_{Q}^{+}(x)\right)^{2}\left(\mu_{R}^{+}(x)\right)^{2}\right)}{\left(\mu_{P}^{+}(x)\right)^{2}+\left(\mu_{Q}^{+}(x)\right)^{2}+2\left(\mu_{R}^{+}(x)\right)^{2}-\left(\mu_{P}^{+}(x)\right)^{2}\left(\mu_{R}^{+}(x)\right)^{2}-\left(\mu_{Q}^{+}(x)\right)^{2}\left(\mu_{R}^{+}(x)\right)^{2}}\right],} \\
& {\left.\left.\left[\frac{\sqrt{2} \nu_{P}^{-}(x) \nu_{Q}^{-}(x) \nu_{R}^{-}(x)}{\sqrt{\left(\nu_{P}^{-}(x)\right)^{2}+\left(\nu_{Q}^{-}(x)\right)^{2}}}, \frac{\sqrt{2} \nu_{P}^{+}(x) \nu_{Q}^{+}(x) \nu_{R}^{+}(x)}{\sqrt{\left(\nu_{P}^{+}(x)\right)^{2}+\left(\nu_{Q}^{+}(x)\right)^{2}}}\right]\right\rangle x \in X\right\} . }
\end{aligned}
$$

\section{Box IX}

$$
\begin{aligned}
f(x)= & \left(\sqrt{\frac{2\left(\mu_{P}^{-}(x)\right)^{2}\left(\mu_{Q}^{-}(x)\right)^{2}+\left(\mu_{P}^{-}(x)\right)^{2}\left(\mu_{R}^{-}(x)\right)^{2}+\left(\mu_{Q}^{-}(x)\right)^{2}\left(\mu_{R}^{-}(x)\right)^{2}-2\left(\mu_{P}^{-}(x)\right)^{2}\left(\mu_{Q}^{-}(x)\right)^{2}\left(\mu_{R}^{-}(x)\right)^{2}}{\left(\mu_{P}^{-}(x)\right)^{2}+\left(\mu_{Q}^{-}(x)\right)^{2}}}\right)^{2} \\
& -\left(\sqrt{\frac{2\left(\left(\mu_{P}^{-}(x)\right)^{2}+\left(\mu_{R}^{-}(x)\right)^{2}-\left(\mu_{P}^{-}(x)\right)^{2}\left(\mu_{R}^{-}(x)\right)^{2}\right)\left(\left(\mu_{Q}^{-}(x)\right)^{2}+\left(\mu_{R}^{-}(x)\right)^{2}-\left(\mu_{Q}^{-}(x)\right)^{2}\left(\mu_{R}^{-}(x)\right)^{2}\right)}{\left(\mu_{P}^{-}(x)\right)^{2}+\left(\mu_{Q}^{-}(x)\right)^{2}+2\left(\mu_{R}^{-}(x)\right)^{2}-\left(\mu_{P}^{-}(x)\right)^{2}\left(\mu_{R}^{-}(x)\right)^{2}-\left(\mu_{Q}^{-}(x)\right)^{2}\left(\mu_{R}^{-}(x)\right)^{2}}}\right)
\end{aligned}
$$

Box X

Definition 11. For every $P \in \operatorname{IVPFS}(X)$ and for every $m, n \in N$, we define:

$$
\begin{aligned}
P^{(m, n)}= & \left\{\left\langlex,\left[\sqrt{\frac{\left(\left(\mu_{P}^{-}(x)\right)^{2}\right)^{m}}{n}}, \sqrt{\frac{\left(\left(\mu_{P}^{+}(x)\right)^{2}\right)^{m}}{n}}\right],\right.\right. \\
& {\left[\sqrt{1-\frac{\left(1-\left(\nu_{P}^{-}(x)\right)^{2}\right)^{m}}{n}},\right.}
\end{aligned}
$$

$$
\left.\left.\sqrt{1-\frac{\left(1-\left(\nu_{P}^{+}(x)\right)^{2}\right)^{m}}{n}}\right]|x \in X\rangle\right\} .
$$

It can be known that $P^{(m, n)}$ is still an IVPFN. From this definition, we can have the following:

(1) If $m \leq m_{1}$, then $P^{(m, n)} \supseteq P^{\left(m_{1}, n\right)}$;

(2) If $n \leq n_{1}$, then $P^{(m, n)} \supseteq P^{\left(m, n_{1}\right)}$;

(3) If $P \subseteq Q$, then $P^{(m, n)} \subseteq Q^{(m, n)}$. 


$$
\begin{aligned}
& f(x)=\frac{2\left(\mu_{P}^{-}(x)\right)^{2}\left(\mu_{Q}^{-}(x)\right)^{2}+\left(\mu_{P}^{-}(x)\right)^{2}\left(\mu_{R}^{-}(x)\right)^{2}+\left(\mu_{Q}^{-}(x)\right)^{2}\left(\mu_{R}^{-}(x)\right)^{2}-2\left(\mu_{P}^{-}(x)\right)^{2}\left(\mu_{Q}^{-}(x)\right)^{2}\left(\mu_{R}^{-}(x)\right)^{2}}{\left(\mu_{P}^{-}(x)\right)^{2}+\left(\mu_{Q}^{-}(x)\right)^{2}} \\
& -\frac{2\left(\left(\mu_{P}^{-}(x)\right)^{2}+\left(\mu_{R}^{-}(x)\right)^{2}-\left(\mu_{P}^{-}(x)\right)^{2}\left(\mu_{R}^{-}(x)\right)^{2}\right)\left(\left(\mu_{Q}^{-}(x)\right)^{2}+\left(\mu_{R}^{-}(x)\right)^{2}-\left(\mu_{Q}^{-}(x)\right)^{2}\left(\mu_{R}^{-}(x)\right)^{2}\right)}{\left(\mu_{P}^{-}(x)\right)^{2}+\left(\mu_{Q}^{-}(x)\right)^{2}+2\left(\mu_{R}^{-}(x)\right)^{2}-\left(\mu_{P}^{-}(x)\right)^{2}\left(\mu_{R}^{-}(x)\right)^{2}-\left(\mu_{Q}^{-}(x)\right)^{2}\left(\mu_{R}^{-}(x)\right)^{2}} \\
& =\frac{1}{\left(\left(\mu_{P}^{-}(x)\right)^{2}+\left(\mu_{Q}^{-}(x)\right)^{2}\right)\left(\left(\mu_{P}^{-}(x)\right)^{2}+\left(\mu_{Q}^{-}(x)\right)^{2}+2\left(\mu_{R}^{-}(x)\right)^{2}-\left(\mu_{P}^{-}(x)\right)^{2}\left(\mu_{R}^{-}(x)\right)^{2}-\left(\mu_{Q}^{-}(x)\right)^{2}\left(\mu_{R}^{-}(x)\right)^{2}\right)} \\
& {\left[\left(2\left(\mu_{P}^{-}(x)\right)^{2}\left(\mu_{Q}^{-}(x)\right)^{2}+\left(\mu_{P}^{-}(x)\right)^{2}\left(\mu_{R}^{-}(x)\right)^{2}+\left(\mu_{Q}^{-}(x)\right)^{2}\left(\mu_{R}^{-}(x)\right)^{2}-2\left(\mu_{P}^{-}(x)\right)^{2}\left(\mu_{Q}^{-}(x)\right)^{2}\left(m u_{R}^{-}(x)\right)^{2}\right)\right.} \\
& \left(\left(\mu_{P}^{-}(x)\right)^{2}+\left(\mu_{Q}^{-}(x)\right)^{2}+\left(\mu_{R}^{-}(x)\right)^{2}+\left(\mu_{R}^{-}(x)\right)^{2}-\left(\mu_{P}^{-}(x)\right)^{2}\left(\mu_{R}^{-}(x)\right)^{2}-\left(\mu_{Q}^{-}(x)\right)^{2}\left(\mu_{R}^{-}(x)\right)^{2}\right) \\
& -2\left(\left(\mu_{P}^{-}(x)\right)^{2}+\left(\mu_{R}^{-}(x)\right)^{2}-\left(\mu_{P}^{-}(x)\right)^{2}\left(\mu_{R}^{-}(x)\right)^{2}\right)\left(\left(\mu_{Q}^{-}(x)\right)^{2}+\left(\mu_{R}^{-}(x)\right)^{2}-\left(\mu_{Q}^{-}(x)\right)^{2}\left(\mu_{R}^{-}(x)\right)^{2}\right) \\
& \left.\left(\left(\mu_{P}^{-}(x)\right)^{2}+\left(\mu_{Q}^{-}(x)\right)^{2}\right)\right] \\
& =\frac{1}{\left(\left(\mu_{P}^{-}(x)\right)^{2}+\left(\mu_{Q}^{-}(x)\right)^{2}\right)\left(\left(\mu_{P}^{-}(x)\right)^{2}+\left(\mu_{Q}^{-}(x)\right)^{2}+2\left(\mu_{R}^{-}(x)\right)^{2}-\left(\mu_{P}^{-}(x)\right)^{2}\left(\mu_{R}^{-}(x)\right)^{2}-\left(\mu_{Q}^{-}(x)\right)^{2}\left(\mu_{R}^{-}(x)\right)^{2}\right)} \\
& {\left[\left(2\left(\mu_{P}^{-}(x)\right)^{2}\left(\mu_{Q}^{-}(x)\right)^{2}+\left(\mu_{P}^{-}(x)\right)^{2}\left(\mu_{R}^{-}(x)\right)^{2}+\left(\mu_{Q}^{-}(x)\right)^{2}\left(\mu_{R}^{-}(x)\right)^{2}-2\left(\mu_{P}^{-}(x)\right)^{2}\left(\mu_{Q}^{-}(x)\right)^{2}\left(\mu_{R}^{-}(x)\right)^{2}\right)\right.} \\
& \left(\left(\mu_{P}^{-}(x)\right)^{2}+\left(\mu_{R}^{-}(x)\right)^{2}-\left(\mu_{P}^{-}(x)\right)^{2}\left(\mu_{R}^{-}(x)\right)^{2}+\left(\mu_{Q}^{-}(x)\right)^{2}+\left(\mu_{R}^{-}(x)\right)^{2}-\left(\mu_{Q}^{-}(x)\right)^{2}\left(\mu_{R}^{-}(x)\right)^{2}\right) \\
& -2\left(\left(\mu_{P}^{-}(x)\right)^{2}+\left(\mu_{R}^{-}(x)\right)^{2}-\left(\mu_{P}^{-}(x)\right)^{2}\left(\mu_{R}^{-}(x)\right)^{2}\right)\left(\left(\mu_{Q}^{-}(x)\right)^{2}+\left(\mu_{R}^{-}(x)\right)^{2}-\left(\mu_{Q}^{-}(x)\right)^{2}\left(\mu_{R}^{-}(x)\right)^{2}\right) \\
& \left.\left(\left(\mu_{P}^{-}(x)\right)^{2}+\left(\mu_{Q}^{-}(x)\right)^{2}\right)\right] \\
& =\frac{1}{\left(\left(\mu_{P}^{-}(x)\right)^{2}+\left(\mu_{Q}^{-}(x)\right)^{2}\right)\left(\left(\mu_{P}^{-}(x)\right)^{2}+\left(\mu_{Q}^{-}(x)\right)^{2}+2\left(\mu_{R}^{-}(x)\right)^{2}-\left(\mu_{P}^{-}(x)\right)^{2}\left(\mu_{R}^{-}(x)\right)^{2}-\left(\mu_{Q}^{-}(x)\right)^{2}\left(\mu_{R}^{-}(x)\right)^{2}\right)} \\
& {\left[\left(2\left(\mu_{P}^{-}(x)\right)^{2}\left(\mu_{Q}^{-}(x)\right)^{2}+\left(\mu_{P}^{-}(x)\right)^{2}\left(\mu_{R}^{-}(x)\right)^{2}+\left(\mu_{Q}^{-}(x)\right)^{2}\left(\mu_{R}^{-}(x)\right)^{2}-2\left(\mu_{P}^{-}(x)\right)^{2}\left(\mu_{Q}^{-}(x)\right)^{2}\left(\mu_{R}^{-}(x)\right)^{2}\right)\right.} \\
& \left(\left(\mu_{P}^{-}(x)\right)^{2}+\left(\mu_{R}^{-}(x)\right)^{2}-\left(\mu_{P}^{-}(x)\right)^{2}\left(\mu_{R}^{-}(x)\right)^{2}\right)+\left(2\left(\mu_{P}^{-}(x)\right)^{2}\left(\mu_{Q}^{-}(x)\right)^{2}+\left(\mu_{P}^{-}(x)\right)^{2}\left(\mu_{R}^{-}(x)\right)^{2}\right. \\
& \left.+\left(\mu_{Q}^{-}(x)\right)^{2}\left(\mu_{R}^{-}(x)\right)^{2}-2\left(\mu_{P}^{-}(x)\right)^{2}\left(\mu_{Q}^{-}(x)\right)^{2}\left(\mu_{R}^{-}(x)\right)^{2}\right)\left(\left(\mu_{Q}^{-}(x)\right)^{2}+\left(\mu_{R}^{-}(x)\right)^{2}-\left(\mu_{Q}^{-}(x)\right)^{2}\left(\mu_{R}^{-}(x)\right)^{2}\right) \\
& -\left(\left(\mu_{P}^{-}(x)\right)^{2}+\left(\mu_{R}^{-}(x)\right)^{2}-\left(\mu_{P}^{-}(x)\right)^{2}\left(\mu_{R}^{-}(x)\right)^{2}\right)\left(\left(\mu_{Q}^{-}(x)\right)^{2}+\left(\mu_{R}^{-}(x)\right)^{2}-\left(\mu_{Q}^{-}(x)\right)^{2}\left(\mu_{R}^{-}(x)\right)^{2}\right) \\
& \left(\left(\mu_{P}^{-}(x)\right)^{2}+\left(\mu_{Q}^{-}(x)\right)^{2}\right)-\left(\left(\mu_{P}^{-}(x)\right)^{2}+\left(\mu_{R}^{-}(x)\right)^{2}-\left(\mu_{P}^{-}(x)\right)^{2}\left(\mu_{R}^{-}(x)\right)^{2}\right) \\
& \left.\left(\left(\mu_{Q}^{-}(x)\right)^{2}+\left(\mu_{R}^{-}(x)\right)^{2}-\left(\mu_{Q}^{-}(x)\right)^{2}\left(\mu_{R}^{-}(x)\right)^{2}\right)\left(\left(\mu_{P}^{-}(x)\right)^{2}+\left(\mu_{Q}^{-}(x)\right)^{2}\right)\right] \\
& =\frac{1}{\left(\left(\mu_{P}^{-}(x)\right)^{2}+\left(\mu_{Q}^{-}(x)\right)^{2}\right)\left(\left(\mu_{P}^{-}(x)\right)^{2}+\left(\mu_{Q}^{-}(x)\right)^{2}+2\left(\mu_{R}^{-}(x)\right)^{2}-\left(\mu_{P}^{-}(x)\right)^{2}\left(\mu_{R}^{-}(x)\right)^{2}-\left(\mu_{Q}^{-}(x)\right)^{2}\left(\mu_{R}^{-}(x)\right)^{2}\right)}
\end{aligned}
$$




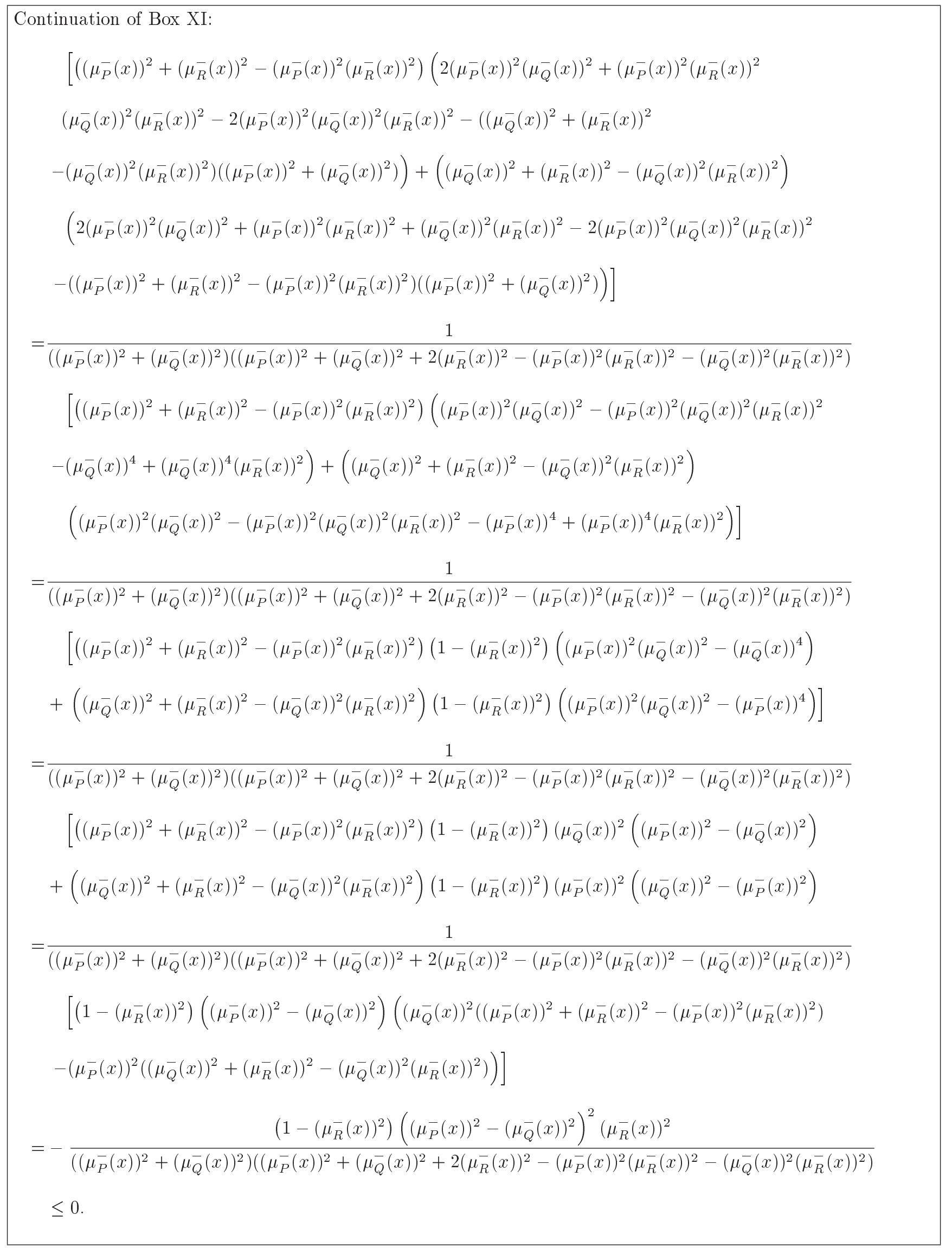




$$
\begin{gathered}
\sqrt{\frac{2\left(\mu_{P}^{-}(x)\right)^{2}\left(\mu_{Q}^{-}(x)\right)^{2}+\left(\mu_{P}^{-}(x)\right)^{2}\left(\mu_{R}^{-}(x)\right)^{2}+\left(\mu_{Q}^{-}(x)\right)^{2}\left(\mu_{R}^{-}(x)\right)^{2}-2\left(\mu_{P}^{-}(x)\right)^{2}\left(\mu_{Q}^{-}(x)\right)^{2}\left(\mu_{R}^{-}(x)\right)^{2}}{\left(\mu_{P}^{-}(x)\right)^{2}+\left(\mu_{Q}^{-}(x)\right)^{2}}} \\
\leq \sqrt{\frac{2\left(\left(\mu_{P}^{-}(x)\right)^{2}+\left(\mu_{R}^{-}(x)\right)^{2}-\left(\mu_{P}^{-}(x)\right)^{2}\left(\mu_{R}^{-}(x)\right)^{2}\right)\left(\left(\mu_{Q}^{-}(x)\right)^{2}+\left(\mu_{R}^{-}(x)\right)^{2}-\left(\mu_{Q}^{-}(x)\right)^{2}\left(\mu_{R}^{-}(x)\right)^{2}\right)}{\left(\mu_{P}^{-}(x)\right)^{2}+\left(\mu_{Q}^{-}(x)\right)^{2}+2\left(\mu_{R}^{-}(x)\right)^{2}-\left(\mu_{P}^{-}(x)\right)^{2}\left(\mu_{R}^{-}(x)\right)^{2}-\left(\mu_{Q}^{-}(x)\right)^{2}\left(\mu_{R}^{-}(x)\right)^{2}}} .
\end{gathered}
$$

Similarly:

$$
\begin{aligned}
& \sqrt{\frac{2\left(\mu_{P}^{+}(x)\right)^{2}\left(\mu_{Q}^{+}(x)\right)^{2}+\left(\mu_{P}^{+}(x)\right)^{2}\left(\mu_{R}^{+}(x)\right)^{2}+\left(\mu_{Q}^{+}(x)\right)^{2}\left(\mu_{R}^{+}(x)\right)^{2}-2\left(\mu_{P}^{+}(x)\right)^{2}\left(\mu_{Q}^{+}(x)\right)^{2}\left(\mu_{R}^{+}(x)\right)^{2}}{\left(\mu_{P}^{+}(x)\right)^{2}+\left(\mu_{Q}^{+}(x)\right)^{2}}} \\
& \leq \sqrt{\frac{2\left(\left(\mu_{P}^{+}(x)\right)^{2}+\left(\mu_{R}^{+}(x)\right)^{2}-\left(\mu_{P}^{+}(x)\right)^{2}\left(\mu_{R}^{+}(x)\right)^{2}\right)\left(\left(\mu_{Q}^{+}(x)\right)^{2}+\left(\mu_{R}^{+}(x)\right)^{2}-\left(\mu_{Q}^{+}(x)\right)^{2}\left(\mu_{R}^{+}(x)\right)^{2}\right)}{\left(\mu_{P}^{+}(x)\right)^{2}+\left(\mu_{Q}^{+}(x)\right)^{2}+2\left(\mu_{R}^{+}(x)\right)^{2}-\left(\mu_{P}^{+}(x)\right)^{2}\left(\mu_{R}^{+}(x)\right)^{2}-\left(\mu_{Q}^{+}(x)\right)^{2}\left(\mu_{R}^{+}(x)\right)^{2}}} .
\end{aligned}
$$

Box XIII

Example 4. Let $P$ be an IVPFS over $X$ such that:

$$
\begin{aligned}
P=\{ & <x_{1},[0.2,0.3],[0.4,0.6]>, \\
& <x_{2},[0.2,0.4],[0.5,0.8]>, \\
& <x_{3},[0.4,0.5],[0.6,0.7]>, \\
& \left.<x_{4},[0.5,0.6],[0.7,0.8]>\right\} .
\end{aligned}
$$

Then, we can easily compute $P^{(m, n)}$ by the above definition shown in Table 2.

For a better understanding of the trend of $P^{(m, n)}$ where $m$ and $n$ are of different values, we give its score function with the figure form shown in Figure 2. Based on Figure 2, we can find that it is decreasing when $m$ (or $n$ ) is increasing when $n$ (or $m$ ) stays at the same level. Meanwhile, when $m$ or $n$ is gradually decreasing, the tread of its score function becomes slight.

Theorem 4. Let $X$ be a nonempty set. For every IVPFS $P$ in $X$ and $m, n \in N$ :

(1) $\left(P^{(m, 1)}\right)^{(n, 1)}=P^{(m n, 1)}=\left(P^{(n, 1)}\right)^{(m, 1)}$;

(2) $\left(P^{(1, m)}\right)^{(1, n)}=P^{(1, m n)}=\left(P^{(1, n)}\right)^{(1, m)}$;

(3) $\quad\left(P^{(m, 1)}\right)^{(1, n)}=P^{(m, n)}$;

(4) $\left(P^{(1, m)}\right)^{(n, 1)}=P^{\left(n, m^{n}\right)}$.

Proof. It is trivial.

Theorem 5. Let $X$ be a nonempty set. For every IVPFS $P$ in $X$ and $m, m_{1}, n, n_{1} \in N$ :

(1) $\square\left(P^{(m, n)}\right)=(\square P)^{(m, n)}$;
(2) $\diamond\left(P^{(m, n)}\right)=(\diamond P)^{(m, n)}$;

(3) $\left(\square\left(P^{(m, n)}\right)\right)^{\left(m_{1}, n_{1}\right)}=\square\left(P^{\left(m m_{1}, n_{1} n^{m_{1}}\right)}\right)$;

(4) $\left(\diamond\left(P^{(m, n)}\right)\right)^{\left(m_{1}, n_{1}\right)}=\diamond\left(P^{\left(m m_{1}, n_{1} n^{m_{1}}\right)}\right)$;

(5) $\left(\square\left(P^{(m, n)}\right)\right)^{\left(m_{1}, n_{1}\right)} \otimes\left(\square\left(P^{(n, m)}\right)\right)^{\left(n_{1}, m_{1}\right)}$ $=\square\left(P^{\left(m m_{1}+n n_{1}, m_{1} n_{1} n^{m_{1}} m^{n_{1}}\right)}\right) ;$

(6) $\left(\diamond\left(P^{(m, n)}\right)\right)^{\left(m_{1}, n_{1}\right)} \otimes\left(\diamond\left(P^{(n, m)}\right)\right)^{\left(n_{1}, m_{1}\right)}$ $=\diamond\left(P^{\left(m m_{1}+n n_{1}, m_{1} n_{1} n^{m_{1}} m^{n_{1}}\right)}\right)$.

Proof. It is trivial.

\subsection{Comparison with new interval-valued Pythagorean fuzzy operators and the existing ones}

3.3.1. Comparison with some existing Pythagorean fuzzy operators

The prominent characteristic of interval-valued Pythagorean fuzzy operators is that they can permit the membership and nonmembership degrees for a given set to have an interval value. This kind of situation is more or less similar to that encountered in intuitionistic fuzzy environments, where the concept of IFSs has been extended to that of interval-valued IFSs to describe the case of interval values in which the membership and nonmembership degrees of an element are assigned to a set. It should be noted that when the upper and lower limits of the interval values are identical, IVPFS becomes PFS, indicating that the latter is a special case of the former. Hence, our proposed interval-valued Pythagorean fuzzy operators are more suitable in solving real problems compared with $[3,5]$. 
Table 2. The results of operation $P^{(m, n)}$ when $m$ and $n$ are with different values.

\begin{tabular}{|c|c|}
\hline$m, n$ & Results \\
\hline$m=1, n=1$ & $\begin{array}{l}\left\{<x_{1},[0.2,0.3],[0.4,0.6]>,<x_{2},[0.2,0.4],[0.5,0.8]>,<x_{3},[0.4,0.5],[0.6,0.7]>\right. \\
\left.\quad<x_{4},[0.5,0.6],[0.7,0.8]>\right\}\end{array}$ \\
\hline$m=1, n=2$ & $\begin{array}{l}\left\{<x_{1},[0.1414,0.2121],[0.7616,0.8246]>,<x_{2},[0.1414,0.2828],[0.7906,0.9055]>\right. \\
\left.\quad<x_{3},[0.2828,0.3536],[0.8246,0.8631]>,<x_{4},[0.3536,0.4243],[0.8631,0.9055]>\right\}\end{array}$ \\
\hline$m=1, n=3$ & $\begin{array}{l}\left\{<x_{1},[0.1155,0.1732],[0.8485,0.8869]>,<x_{2},[0.1155,0.2309],[0.8660,0.9381]>\right. \\
\left.\quad<x_{3},[0.2309,0.2887],[0.8869,0.9110]>,<x_{4},[0.2887,0.3464],[0.9110,0.9381]>\right\}\end{array}$ \\
\hline$m=1, n=4$ & $\begin{array}{l}\left\{<x_{1},[0.1000,0.1500],[0.8888,0.9165]>,<x_{2},[0.1000,0.2000],[0.9014,0.9539]>\right. \\
\left.\quad<x_{3},[0.2000,0.2500],[0.9165,0.9341]>,<x_{4},[0.2500,0.3000],[0.9341,0.9539]>\right\}\end{array}$ \\
\hline$m=1, n=5$ & $\begin{array}{l}\left\{<x_{1},[0.0894,0.1342],[0.9121,0.9338]>,<x_{2},[0.0894,0.1789],[0.9220,0.9633]>\right. \\
\left.\quad<x_{3},[0.1789,0.2236],[0.9338,0.9476]>,<x_{4},[0.2236,0.2683],[0.9476,0.9633]>\right\}\end{array}$ \\
\hline$m=1, n=6$ & $\begin{array}{l}\left\{<x_{1},[0.0816,0.1225],[0.9274,0.9452]>,<x_{2},[0.0816,0.1633],[0.9354,0.9695]>\right. \\
\left.\quad<x_{3},[0.1633,0.2041],[0.9452,0.9566]>,<x_{4},[0.2041,0.2449],[0.9566,0.9695]>\right\}\end{array}$ \\
\hline$m=1, n=7$ & $\begin{array}{l}\left\{<x_{1},[0.0756,0.1134],[0.9381,0.9532]>,<x_{2},[0.0756,0.1512],[0.9449,0.9739]>\right. \\
\left.\quad<x_{3},[0.1512,0.1890],[0.9532,0.9629]>,<x_{4},[0.1890,0.2268],[0.9629,0.9739]>\right\}\end{array}$ \\
\hline$m=1, n=8$ & $\begin{array}{l}\left\{<x_{1},[0.0707,0.1061],[0.9460,0.9592]>,<x_{2},[0.0707,0.1414],[0.9520,0.9772]>\right. \\
\left.\quad<x_{3},[0.1414,0.1768],[0.9592,0.9676]>,<x_{4},[0.1768,0.2121],[0.9676,0.9772]>\right\}\end{array}$ \\
\hline$m=1, n=9$ & $\begin{array}{l}\left\{<x_{1},[0.0667,0.1000],[0.9522,0.9638]>,<x_{2},[0.0667,0.1333],[0.9574,0.9798]>\right. \\
\left.\quad<x_{3},[0.1333,0.1667],[0.9638,0.9713]>,<x_{4},[0.1667,0.2000],[0.9713,0.9798]>\right\}\end{array}$ \\
\hline$m=1, n=10$ & $\begin{array}{l}\left\{<x_{1},[0.0632,0.0949],[0.9571,0.9675]>,<x_{2},[0.0632,0.1265],[0.9618,0.9818]>\right. \\
\left.\quad<x_{3},[0.1265,0.1581],[0.9675,0.9742]>,<x_{4},[0.1581,0.1897],[0.9742,0.9818]>\right\}\end{array}$ \\
\hline$m=2, n=1$ & $\begin{array}{l}\left\{<x_{1},[0.0400,0.0900],[0.5426,0.7684]>,<x_{2},[0.0400,0.1600],[0.6614,0.9330]>\right. \\
\left.<x_{3},[0.1600,0.2500],[0.7684,0.8602]>,<x_{4},[0.2500,0.3600],[0.8602,0.9330]>\right\}\end{array}$ \\
\hline$m=2, n=2$ & $\begin{array}{l}\left\{<x_{1},[0.0283,0.0636],[0.8045,0.8917]>,<x_{2},[0.0283,0.1131],[0.8478,0.9671]>\right. \\
\left.\quad<x_{3},[0.1131,0.1768],[0.8917,0.9327]>,<x_{4},[0.1768,0.2546],[0.9327,0.9671]>\right\}\end{array}$ \\
\hline$m=2, n=3$ & $\begin{array}{r}\left\{<x_{1},[0.0231,0.0520],[0.8745,0.9292]>,<x_{2},[0.0231,0.0924],[0.9014,0.9782]>\right. \\
\left.\quad<x_{3},[0.0924,0.1443],[0.9292,0.9557]>,<x_{4},[0.1443,0.2078],[0.9557,0.9782]>\right\}\end{array}$ \\
\hline$m=2, n=4$ & $\begin{array}{l}\left\{<x_{1},[0.0200,0.0450],[0.9075,0.9474]>,<x_{2},[0.0200,0.0800],[0.9270,0.9837]>\right. \\
\left.\quad<x_{3},[0.0800,0.1250],[0.9474,0.9669]>,<x_{4},[0.1250,0.1800],[0.9669,0.9837]>\right\}\end{array}$ \\
\hline$m=2, n=5$ & $\begin{array}{l}\left\{<x_{1},[0.0179,0.0402],[0.9268,0.9582]>,<x_{2},[0.0179,0.0716],[0.9421,0.9870]>\right. \\
\left.\quad<x_{3},[0.0716,0.1118],[0.9582,0.9736]>,<x_{4},[0.1118,0.1610],[0.9736,0.9870]>\right\}\end{array}$ \\
\hline$m=2, n=6$ & $\begin{array}{l}\left\{<x_{1},[0.0163,0.0367],[0.9394,0.9653]>,<x_{2},[0.0163,0.0653],[0.9520,0.9891]>\right. \\
\left.\quad<x_{3},[0.0653,0.1021],[0.9653,0.9781]>,<x_{4},[0.1021,0.1470],[0.9781,0.9891]>\right\}\end{array}$ \\
\hline
\end{tabular}


Table 2. The results of operation $P^{(m, n)}$ when $m$ and $n$ are with different values (continued).

\begin{tabular}{|c|c|}
\hline$m, n$ & Results \\
\hline$m=2, n=7$ & $\begin{aligned}\{ & <x_{1},[0.0151,0.0340],[0.9483,0.9703]>,<x_{2},[0.0151,0.0605],[0.9590,0.9907]> \\
& \left.<x_{3},[0.0605,0.0945],[0.9703,0.9812]>,<x_{4},[0.0945,0.1361],[0.9812,0.9907]>\right\}\end{aligned}$ \\
\hline$m=2, n=8$ & $\begin{aligned}\left\{<x_{1},[0.0141,0.0318],[0.9549,0.9741]>,<x_{2},[0.0141,0.0566],[0.9642,0.9919]>\right. \\
\left.<x_{3},[0.0566,0.0884],[0.9741,0.9836]>,<x_{4},[0.0884,0.1273],[0.9836,0.9919]>\right\}\end{aligned}$ \\
\hline$m=2, n=9$ & $\begin{array}{l}\left\{<x_{1},[0.0133,0.0300],[0.9600,0.9770]>,<x_{2},[0.0133,0.0533],[0.9682,0.9928]>\right. \\
\left.<x_{3},[0.0533,0.0833],[0.9770,0.9854]>,<x_{4},[0.0833,0.1200],[0.9854,0.9928]>\right\}\end{array}$ \\
\hline$m=2, n=10$ & $\begin{aligned}\{ & <x_{1},[0.0126,0.0285],[0.9641,0.9793]>,<x_{2},[0.0126,0.0506],[0.9715,0.9935]> \\
& \left.<x_{3},[0.0506,0.0791],[0.9793,0.9869]>,<x_{4},[0.0791,0.1138],[0.9869,0.9935]>\right\}\end{aligned}$ \\
\hline$m=3, n=1$ & $\begin{aligned}\{ & <x_{1},[0.0080,0.0270],[0.6382,0.8590]>,<x_{2},[0.0080,0.0640],[0.7603,0.9764]> \\
& \left.<x_{3},[0.0640,0.1250],[0.8590,0.9313]>,<x_{4},[0.1250,0.2160],[0.9313,0.9764]>\right\}\end{aligned}$ \\
\hline$m=3, n=2$ & $\begin{array}{l}\left\{<x_{1},[0.0057,0.0191],[0.8388,0.9322]>,<x_{2},[0.0057,0.0453],[0.8883,0.9883]>\right. \\
\left.\quad<x_{3},[0.0453,0.0884],[0.9322,0.9663]>,<x_{4},[0.0884,0.1527],[0.9663,0.9883]>\right\}\end{array}$ \\
\hline$m=3, n=3$ & $\begin{array}{l}\left\{<x_{1},[0.0046,0.0156],[0.8958,0.9553]>,<x_{2},[0.0046,0.0370],[0.9270,0.9922]>\right. \\
\left.<x_{3},[0.0370,0.0722],[0.9553,0.9776]>,<x_{4},[0.0722,0.1247],[0.9776,0.9922]>\right\}\end{array}$ \\
\hline$m=3, n=4$ & $\begin{array}{l}\left\{<x_{1},[0.0040,0.0135],[0.9229,0.9667]>,<x_{2},[0.0040,0.0320],[0.9458,0.9942]>\right. \\
\left.<x_{3},[0.0320,0.0625],[0.9667,0.9833]>,<x_{4},[0.0625,0.1080],[0.9833,0.9942]>\right\}\end{array}$ \\
\hline$m=3, n=5$ & $\begin{array}{l}\left\{<x_{1},[0.0036,0.0121],[0.9389,0.9734]>,<x_{2},[0.0036,0.0286],[0.9569,0.9953]>\right. \\
\left.<x_{3},[0.0286,0.0559],[0.9734,0.9866]>,<x_{4},[0.0559,0.0966],[0.9866,0.9953]>\right\}\end{array}$ \\
\hline$m=3, n=6$ & $\begin{array}{l}\left\{<x_{1},[0.0033,0.0110],[0.9493,0.9779]>,<x_{2},[0.0033,0.0261],[0.9642,0.9961]>\right. \\
\left.<x_{3},[0.0261,0.0510],[0.9779,0.9889]>,<x_{4},[0.0510,0.0882],[0.9889,0.9961]>\right\}\end{array}$ \\
\hline$m=3, n=7$ & $\begin{array}{l}\left\{<x_{1},[0.0030,0.0102],[0.9567,0.9811]>,<x_{2},[0.0030,0.0242],[0.9694,0.9967]>\right. \\
\left.<x_{3},[0.0242,0.0472],[0.9811,0.9905]>,<x_{4},[0.0472,0.0816],[0.9905,0.9967]>\right\}\end{array}$ \\
\hline$m=3, n=8$ & $\begin{array}{l}\left\{<x_{1},[0.0028,0.0095],[0.9622,0.9835]>,<x_{2},[0.0028,0.0226],[0.9733,0.9971]>\right. \\
\left.<x_{3},[0.0226,0.0442],[0.9835,0.9917]>,<x_{4},[0.0442,0.0764],[0.9917,0.9971]>\right\}\end{array}$ \\
\hline$m=3, n=9$ & $\begin{aligned} &\left\{<x_{1},[0.0027,0.0090],[0.9665,0.9853]>,<x_{2},[0.0027,0.0213],[0.9763,0.9974]>\right. \\
&<x_{3},[0.0213,0.0417],[0.9853,0.9926]>\left.<x_{4},[0.0417,0.0720],[0.9926,0.9974]>\right\}\end{aligned}$ \\
\hline$m=3, n=10$ & $\begin{array}{l}\left\{<x_{1},[0.0025,0.0085],[0.9699,0.9868]>,<x_{2},[0.0025,0.0202],[0.9787,0.9977]>\right. \\
\left.\quad<x_{3},[0.0202,0.0395],[0.9868,0.9933]>,<x_{4},[0.0395,0.0683],[0.9933,0.9977]>\right\}\end{array}$ \\
\hline$m=4, n=1$ & $\begin{array}{r}\left\{<x_{1},[0.0016,0.0081],[0.7086,0.9123]>,<x_{2},[0.0016,0.0256],[0.8268,0.9916]>\right. \\
\left.\quad<x_{3},[0.0256,0.0625],[0.9123,0.9656]>,<x_{4},[0.0625,0.1296],[0.9656,0.9916]>\right\}\end{array}$ \\
\hline
\end{tabular}


Table 2. The results of operation $P^{(m, n)}$ when $m$ and $n$ are with different values (continued).

\begin{tabular}{|c|c|}
\hline$m, n$ & Results \\
\hline$m=4, n=2$ & $\begin{array}{l}\left\{<x_{1},[0.0011,0.0057],[0.8666,0.9571]>,<x_{2},[0.0011,0.0181],[0.9175,0.9958]>\right. \\
\left.<x_{3},[0.0181,0.0442],[0.9571,0.9829]>,<x_{4},[0.0442,0.0916],[0.9829,0.9958]>\right\}\end{array}$ \\
\hline$m=4, n=3$ & $\begin{array}{l}\left\{<x_{1},[0.0009,0.0047],[0.9133,0.9716]>,<x_{2},[0.0009,0.0148],[0.9458,0.9972]>\right. \\
\left.<x_{3},[0.0148,0.0361],[0.9716,0.9887]>,<x_{4},[0.0361,0.0748],[0.9887,0.9972]>\right\}\end{array}$ \\
\hline$m=4, n=4$ & $\begin{aligned}\left\{<x_{1},[0.0008,0.0041],[0.9357,0.9788]>,<x_{2},[0.0008,0.0128],[0.9596,0.9979]>\right. \\
\left.<x_{3},[0.0128,0.0313],[0.9788,0.9915]>,<x_{4},[0.0313,0.0648],[0.9915,0.9979]>\right\}\end{aligned}$ \\
\hline$m=4, n=5$ & $\begin{array}{r}\left\{<x_{1},[0.0007,0.0036],[0.9489,0.9831]>,<x_{2},[0.0007,0.0114],[0.9678,0.9983]>\right. \\
\left.<x_{3},[0.0114,0.0280],[0.9831,0.9932]>,<x_{4},[0.0280,0.0580],[0.9932,0.9983]>\right\}\end{array}$ \\
\hline$m=4, n=6$ & $\begin{array}{l}\left\{<x_{1},[0.0007,0.0033],[0.9576,0.9859]>,<x_{2},[0.0007,0.0105],[0.9733,0.9986]>\right. \\
\left.<x_{3},[0.0105,0.0255],[0.9859,0.9943]>,<x_{4},[0.0255,0.0529],[0.9943,0.9986]>\right\}\end{array}$ \\
\hline$m=4, n=7$ & $\begin{array}{l}\left\{<x_{1},[0.0006,0.0031],[0.9638,0.9879]>,<x_{2},[0.0006,0.0097],[0.9771,0.9988]>\right. \\
\left.<x_{3},[0.0097,0.0236],[0.9879,0.9952]>,<x_{4},[0.0236,0.0490],[0.9952,0.9988]>\right\}\end{array}$ \\
\hline$m=4, n=8$ & $\begin{aligned}\{ & <x_{1},[0.0006,0.0029],[0.9684,0.9895]>,<x_{2},[0.0006,0.0091],[0.9800,0.9989]> \\
& \left.<x_{3},[0.0091,0.0221],[0.9895,0.9958]>,<x_{4},[0.0221,0.0458],[0.9958,0.9989]>\right\}\end{aligned}$ \\
\hline$m=4, n=9$ & $\begin{aligned}\{ & <x_{1},[0.0005,0.0027],[0.9719,0.9906]>,<x_{2},[0.0005,0.0085],[0.9823,0.9991]> \\
& \left.<x_{3},[0.0085,0.0208],[0.9906,0.9962]>,<x_{4},[0.0208,0.0432],[0.9962,0.9991]>\right\}\end{aligned}$ \\
\hline$m=4, n=10$ & $\begin{aligned}\{ & <x_{1},[0.0005,0.0026],[0.9748,0.9916]>,<x_{2},[0.0005,0.0081],[0.9841,0.9992]> \\
& \left.<x_{3},[0.0081,0.0198],[0.9916,0.9966]>,<x_{4},[0.0198,0.0410],[0.9966,0.9992]>\right\}\end{aligned}$ \\
\hline$m=5, n=1$ & $\begin{array}{l}\left\{<x_{1},[0.0003,0.0024],[0.7628,0.9448]>,<x_{2},[0.0003,0.0102],[0.8733,0.9970]>\right. \\
\left.<x_{3},[0.0102,0.0313],[0.9448,0.9826]>,<x_{4},[0.0313,0.0778],[0.9826,0.9970]>\right\}\end{array}$ \\
\hline$m=5, n=2$ & $\begin{aligned}\{ & <x_{1},[0.0002,0.0017],[0.8893,0.9728]>,<x_{2},[0.0002,0.0072],[0.9388,0.9985]> \\
& \left.<x_{3},[0.0072,0.0221],[0.9728,0.9913]>,<x_{4},[0.0221,0.0550],[0.9913,0.9985]>\right\}\end{aligned}$ \\
\hline$m=5, n=3$ & $\begin{aligned}\{ & <x_{1},[0.0002,0.0014],[0.9277,0.9819]>,<x_{2},[0.0002,0.0059],[0.9596,0.9990]> \\
& \left.<x_{3},[0.0059,0.0180],[0.9819,0.9942]>,<x_{4},[0.0180,0.0449],[0.9942,0.9990]>\right\}\end{aligned}$ \\
\hline$m=5, n=4$ & $\begin{array}{l}\left\{<x_{1},[0.0002,0.0012],[0.9463,0.9865]>,<x_{2},[0.0002,0.0051],[0.9699,0.9992]>\right. \\
\left.<x_{3},[0.0051,0.0156],[0.9865,0.9957]>,<x_{4},[0.0156,0.0389],[0.9957,0.9992]>\right\}\end{array}$ \\
\hline$m=5, n=5$ & $\begin{array}{l}\left\{<x_{1},[0.0001,0.0011],[0.9573,0.9892]>,<x_{2},[0.0001,0.0046],[0.9760,0.9994]>\right. \\
\left.<x_{3},[0.0046,0.0140],[0.9892,0.9965]>,<x_{4},[0.0140,0.0348],[0.9965,0.9994]>\right\}\end{array}$ \\
\hline$m=5, n=6$ & $\begin{array}{r}\left\{<x_{1},[0.0001,0.0010],[0.9645,0.9910]>,<x_{2},[0.0001,0.0042],[0.9800,0.9995]>\right. \\
\left.<x_{3},[0.0042,0.0128],[0.9910,0.9971]>,<x_{4},[0.0128,0.0317],[0.9971,0.9995]>\right\}\end{array}$ \\
\hline
\end{tabular}


Table 2. The results of operation $P^{(m, n)}$ when $m$ and $n$ are with different values (continued).

\begin{tabular}{|c|c|}
\hline$m, n$ & Results \\
\hline$m=5, n=7$ & $\begin{aligned}\{ & <x_{1},[0.0001,0.0009],[0.9697,0.9923]>,<x_{2},[0.0001,0.0039],[0.9829,0.9996]> \\
& \left.<x_{3},[0.0039,0.0118],[0.9923,0.9975]>,<x_{4},[0.0118,0.0294],[0.9975,0.9996]>\right\}\end{aligned}$ \\
\hline$m=5, n=8$ & $\begin{aligned}\left\{<x_{1},[0.0001,0.0009],[0.9735,0.9933]>,<x_{2},[0.0001,0.0036],[0.9851,0.9996]>\right. \\
\left.<x_{3},[0.0036,0.0110],[0.9933,0.9978]>,<x_{4},[0.0110,0.0275],[0.9978,0.9996]>\right\}\end{aligned}$ \\
\hline$m=5, n=9$ & $\begin{aligned}\left\{<x_{1},[0.0001,0.0008],[0.9765,0.9940]>,<x_{2},[0.0001,0.0034],[0.9867,0.9997]>\right. \\
\left.<x_{3},[0.0034,0.0104],[0.9940,0.9981]>,<x_{4},[0.0104,0.0259],[0.9981,0.9997]>\right\}\end{aligned}$ \\
\hline$m=5, n=10$ & $\begin{aligned}\left\{<x_{1},[0.0001,0.0008],[0.9789,0.9946]>,<x_{2},[0.0001,0.0032],[0.9881,0.9997]>\right. \\
\left.<x_{3},[0.0032,0.0099],[0.9946,0.9983]>,<x_{4},[0.0099,0.0246],[0.9983,0.9997]>\right\}\end{aligned}$ \\
\hline$m=6, n=1$ & $\begin{aligned}\{ & <x_{1},[0.0001,0.0007],[0.8054,0.9650]>,<x_{2},[0.0001,0.0041],[0.9067,0.9989]> \\
& \left.<x_{3},[0.0041,0.0156],[0.9650,0.9912]>,<x_{4},[0.0156,0.0467],[0.9912,0.9989]>\right\}\end{aligned}$ \\
\hline$m=6, n=2$ & $\begin{aligned}\{ & <x_{1},[0.0000,0.0005],[0.9079,0.9827]>,<x_{2},[0.0000,0.0029],[0.9545,0.9995]> \\
& \left.<x_{3},[0.0029,0.0110],[0.9827,0.9956]>,<x_{4},[0.0110,0.0330],[0.9956,0.9995]>\right\}\end{aligned}$ \\
\hline$m=6, n=3$ & $\begin{array}{l}\left\{<x_{1},[0.0000,0.0004],[0.9396,0.9885]>,<x_{2},[0.0000,0.0024],[0.9699,0.9996]>\right. \\
\left.<x_{3},[0.0024,0.0090],[0.9885,0.9971]>,<x_{4},[0.0090,0.0269],[0.9971,0.9996]>\right\}\end{array}$ \\
\hline$m=6, n=4$ & $\begin{aligned}\left\{<x_{1},[0.0000,0.0004],[0.9551,0.9914]>,<x_{2},[0.0000,0.0020],[0.9775,0.9997]>\right. \\
\left.<x_{3},[0.0020,0.0078],[0.9914,0.9978]>,<x_{4},[0.0078,0.0233],[0.9978,0.9997]>\right\}\end{aligned}$ \\
\hline$m=6, n=5$ & $\begin{array}{l}\left\{<x_{1},[0.0000,0.0003],[0.9642,0.9931]>,<x_{2},[0.0000,0.0018],[0.9820,0.9998]>\right. \\
\left.\quad<x_{3},[0.0018,0.0070],[0.9931,0.9982]>,<x_{4},[0.0070,0.0209],[0.9982,0.9998]>\right\}\end{array}$ \\
\hline$m=6, n=6$ & $\begin{array}{l}\left\{<x_{1},[0.0000,0.0003],[0.9703,0.9943]>,<x_{2},[0.0000,0.0017],[0.9851,0.9998]>\right. \\
\left.<x_{3},[0.0017,0.0064],[0.9943,0.9985]>,<x_{4},[0.0064,0.0190],[0.9985,0.9998]>\right\}\end{array}$ \\
\hline$m=6, n=7$ & $\begin{array}{l}\left\{<x_{1},[0.0000,0.0003],[0.9746,0.9951]>,<x_{2},[0.0000,0.0015],[0.9872,0.9998]>\right. \\
\left.<x_{3},[0.0015,0.0059],[0.9951,0.9987]>,<x_{4},[0.0059,0.0176],[0.9987,0.9998]>\right\}\end{array}$ \\
\hline$m=6, n=8$ & $\begin{array}{l}\left\{<x_{1},[0.0000,0.0003],[0.9778,0.9957]>,<x_{2},[0.0000,0.0014],[0.9888,0.9999]>\right. \\
\left.<x_{3},[0.0014,0.0055],[0.9957,0.9989]>,<x_{4},[0.0055,0.0165],[0.9989,0.9999]>\right\}\end{array}$ \\
\hline$m=6, n=9$ & $\begin{aligned}\left\{<x_{1},[0.0000,0.0002],[0.9803,0.9962]>,<x_{2},[0.0000,0.0014],[0.9901,0.9999]>\right. \\
\left.<x_{3},[0.0014,0.0052],[0.9962,0.9990]><x_{4},[0.0052,0.0156],[0.9990,0.9999]>\right\}\end{aligned}$ \\
\hline$m=6, n=10$ & $\begin{aligned}\{ & <x_{1},[0.0000,0.0002],[0.9823,0.9966]>,<x_{2},[0.0000,0.0013],[0.9911,0.9999]> \\
& \left.<x_{3},[0.0013,0.0049],[0.9966,0.9991]>,<x_{4},[0.0049,0.0148],[0.9991,0.9999]>\right\}\end{aligned}$ \\
\hline$m=7, n=1$ & $\begin{array}{r}\left\{<x_{1},[0.0000,0.0002],[0.8396,0.9778]>,<x_{2},[0.0000,0.0016],[0.9309,0.9996]>\right. \\
\left.<x_{3},[0.0016,0.0078],[0.9778,0.9955]>,<x_{4},[0.0078,0.0280],[0.9955,0.9996]>\right\}\end{array}$ \\
\hline
\end{tabular}


Table 2. The results of operation $P^{(m, n)}$ when $m$ and $n$ are with different values (continued).

\begin{tabular}{|c|c|}
\hline$m, n$ & Results \\
\hline$m=7, n=2$ & $\begin{array}{l}\left\{<x_{1},[0.0000,0.0002],[0.9233,0.9889]>,<x_{2},[0.0000,0.0012],[0.9661,0.9998]>\right. \\
\left.<x_{3},[0.0012,0.0055],[0.9889,0.9978]>,<x_{4},[0.0055,0.0198],[0.9978,0.9998]>\right\}\end{array}$ \\
\hline$m=7, n=3$ & $\begin{array}{l}\left\{<x_{1},[0.0000,0.0001],[0.9495,0.9926]>,<x_{2},[0.0000,0.0009],[0.9775,0.9999]>\right. \\
\left.<x_{3},[0.0009,0.0045],[0.9926,0.9985]>,<x_{4},[0.0045,0.0162],[0.9985,0.9999]>\right\}\end{array}$ \\
\hline$m=7, n=4$ & $\begin{array}{l}\left\{<x_{1},[0.0000,0.0001],[0.9624,0.9945]>,<x_{2},[0.0000,0.0008],[0.9832,0.9999]>\right. \\
\left.<x_{3},[0.0008,0.0039],[0.9945,0.9989]>,<x_{4},[0.0039,0.0140],[0.9989,0.9999]>\right\}\end{array}$ \\
\hline$m=7, n=5$ & $\begin{array}{r}\left\{<x_{1},[0.0000,0.0001],[0.9700,0.9956]>,<x_{2},[0.0000,0.0007],[0.9866,0.9999]>\right. \\
\left.<x_{3},[0.0007,0.0035],[0.9956,0.9991]>,<x_{4},[0.0035,0.0125],[0.9991,0.9999]>\right\}\end{array}$ \\
\hline$m=7, n=6$ & $\begin{array}{l}\left\{<x_{1},[0.0000,0.0001],[0.9751,0.9963]>,<x_{2},[0.0000,0.0007],[0.9888,0.9999]>\right. \\
\left.<x_{3},[0.0007,0.0032],[0.9963,0.9993]>,<x_{4},[0.0032,0.0114],[0.9993,0.9999]>\right\}\end{array}$ \\
\hline$m=7, n=7$ & $\begin{array}{l}\left\{<x_{1},[0.0000,0.0001],[0.9787,0.9969]>,<x_{2},[0.0000,0.0006],[0.9904,0.9999]>\right. \\
\left.<x_{3},[0.0006,0.0030],[0.9969,0.9994]>,<x_{4},[0.0030,0.0106],[0.9994,0.9999]>\right\}\end{array}$ \\
\hline$m=7, n=8$ & $\begin{aligned}\{ & <x_{1},[0.0000,0.0001],[0.9814,0.9972]>,<x_{2},[0.0000,0.0006],[0.9916,1.0000]> \\
& \left.<x_{3},[0.0006,0.0028],[0.9972,0.9994]>,<x_{4},[0.0028,0.0099],[0.9994,1.0000]>\right\}\end{aligned}$ \\
\hline$m=7, n=9$ & $\begin{aligned}\{ & <x_{1},[0.0000,0.0001],[0.9835,0.9976]>,<x_{2},[0.0000,0.0005],[0.9926,1.0000]> \\
& \left.<x_{3},[0.0005,0.0026],[0.9976,0.9995]>,<x_{4},[0.0026,0.0093],[0.9995,1.0000]>\right\}\end{aligned}$ \\
\hline$m=7, n=10$ & $\begin{aligned}\{ & <x_{1},[0.0000,0.0001],[0.9851,0.9978]>,<x_{2},[0.0000,0.0005],[0.9933,1.0000]> \\
& \left.<x_{3},[0.0005,0.0025],[0.9978,0.9996]>,<x_{4},[0.0025,0.0089],[0.9996,1.0000]>\right\}\end{aligned}$ \\
\hline$m=8, n=1$ & $\begin{aligned}\{ & <x_{1},[0.0000,0.0001],[0.8673,0.9858]>,<x_{2},[0.0000,0.0007],[0.9486,0.9999]> \\
& \left.<x_{3},[0.0007,0.0039],[0.9858,0.9977]>,<x_{4},[0.0039,0.0168],[0.9977,0.9999]>\right\}\end{aligned}$ \\
\hline$m=8, n=2$ & $\begin{aligned}\{ & <x_{1},[0.0000,0.0000],[0.9360,0.9929]>,<x_{2},[0.0000,0.0005],[0.9747,0.9999]> \\
& \left.<x_{3},[0.0005,0.0028],[0.9929,0.9989]>,<x_{4},[0.0028,0.0119],[0.9989,0.9999]>\right\}\end{aligned}$ \\
\hline$m=8, n=3$ & $\begin{aligned}\{ & <x_{1},[0.0000,0.0000],[0.9578,0.9953]>,<x_{2},[0.0000,0.0004],[0.9832,1.0000]> \\
& \left.<x_{3},[0.0004,0.0023],[0.9953,0.9992]>,<x_{4},[0.0023,0.0097],[0.9992,1.0000]>\right\}\end{aligned}$ \\
\hline$m=8, n=4$ & $\begin{array}{l}\left\{<x_{1},[0.0000,0.0000],[0.9685,0.9965]>,<x_{2},[0.0000,0.0003],[0.9874,1.0000]>\right. \\
\left.<x_{3},[0.0003,0.0020],[0.9965,0.9994]>,<x_{4},[0.0020,0.0084],[0.9994,1.0000]>\right\}\end{array}$ \\
\hline$m=8, n=5$ & $\begin{array}{r}\left\{<x_{1},[0.0000,0.0000],[0.9749,0.9972]>,<x_{2},[0.0000,0.0003],[0.9899,1.0000]>\right. \\
\left.<x_{3},[0.0003,0.0017],[0.9972,0.9995]>,<x_{4},[0.0017,0.0075],[0.9995,1.0000]>\right\}\end{array}$ \\
\hline$m=8, n=6$ & $\begin{array}{r}\left\{<x_{1},[0.0000,0.0000],[0.9791,0.9977]>,<x_{2},[0.0000,0.0003],[0.9916,1.0000]>\right. \\
\left.<x_{3},[0.0003,0.0016],[0.9977,0.9996]>,<x_{4},[0.0016,0.0069],[0.9996,1.0000]>\right\}\end{array}$ \\
\hline
\end{tabular}


Table 2. The results of operation $P^{(m, n)}$ when $m$ and $n$ are with different values (continued).

\begin{tabular}{|c|c|}
\hline$m, n$ & Results \\
\hline$m=8, n=7$ & $\begin{array}{r}\left\{<x_{1},[0.0000,0.0000],[0.9821,0.9980]>,<x_{2},[0.0000,0.0002],[0.9928,1.0000]>\right. \\
\left.<x_{3},[0.0002,0.0015],[0.9980,0.9997]>,<x_{4},[0.0015,0.0063],[0.9997,1.0000]>\right\}\end{array}$ \\
\hline$m=8, n=8$ & $\begin{array}{l}\left\{<x_{1},[0.0000,0.0000],[0.9844,0.9982]>,<x_{2},[0.0000,0.0002],[0.9937,1.0000]>\right. \\
\left.<x_{3},[0.0002,0.0014],[0.9982,0.9997]>,<x_{4},[0.0014,0.0059],[0.9997,1.0000]>\right\}\end{array}$ \\
\hline$m=8, n=9$ & $\begin{aligned}\{ & <x_{1},[0.0000,0.0000],[0.9861,0.9984]>,<x_{2},[0.0000,0.0002],[0.9944,1.0000]> \\
& \left.<x_{3},[0.0002,0.0013],[0.9984,0.9997]>,<x_{4},[0.0013,0.0056],[0.9997,1.0000]>\right\}\end{aligned}$ \\
\hline$m=8, n=10$ & $\begin{array}{r}\left\{<x_{1},[0.0000,0.0000],[0.9875,0.9986]>,<x_{2},[0.0000,0.0002],[0.9950,1.0000]>\right. \\
\left.<x_{3},[0.0002,0.0012],[0.9986,0.9998]>,<x_{4},[0.0012,0.0053],[0.9998,1.0000]>\right\}\end{array}$ \\
\hline$m=9, n=1$ & $\begin{aligned}\{ & <x_{1},[0.0000,0.0000],[0.8898,0.9910]>,<x_{2},[0.0000,0.0003],[0.9617,0.9999]> \\
& \left.<x_{3},[0.0003,0.0020],[0.9910,0.9988]>,<x_{4},[0.0020,0.0101],[0.9988,0.9999]>\right\}\end{aligned}$ \\
\hline$m=9, n=2$ & $\begin{aligned}\{ & <x_{1},[0.0000,0.0000],[0.9465,0.9955]>,<x_{2},[0.0000,0.0002],[0.9810,1.0000]> \\
& \left.<x_{3},[0.0002,0.0014],[0.9955,0.9994]>,<x_{4},[0.0014,0.0071],[0.9994,1.0000]>\right\}\end{aligned}$ \\
\hline$m=9, n=3$ & $\begin{aligned}\{ & <x_{1},[0.0000,0.0000],[0.9647,0.9970]>,<x_{2},[0.0000,0.0002],[0.9874,1.0000]> \\
& \left.<x_{3},[0.0002,0.0011],[0.9970,0.9996]>,<x_{4},[0.0011,0.0058],[0.9996,1.0000]>\right\}\end{aligned}$ \\
\hline$m=9, n=4$ & $\begin{aligned}\{ & <x_{1},[0.0000,0.0000],[0.9736,0.9977]>,<x_{2},[0.0000,0.0001],[0.9906,1.0000]> \\
& \left.<x_{3},[0.0001,0.0010],[0.9977,0.9997]>,<x_{4},[0.0010,0.0050],[0.9997,1.0000]>\right\}\end{aligned}$ \\
\hline$m=9, n=5$ & $\begin{aligned}\{ & <x_{1},[0.0000,0.0000],[0.9790,0.9982]>,<x_{2},[0.0000,0.0001],[0.9925,1.0000]> \\
& \left.<x_{3},[0.0001,0.0009],[0.9982,0.9998]>,<x_{4},[0.0009,0.0045],[0.9998,1.0000]>\right\}\end{aligned}$ \\
\hline$m=9, n=6$ & $\begin{array}{l}\left\{<x_{1},[0.0000,0.0000],[0.9825,0.9985]>,<x_{2},[0.0000,0.0001],[0.9937,1.0000]>\right. \\
\left.<x_{3},[0.0001,0.0008],[0.9985,0.9998]>,<x_{4},[0.0008,0.0041],[0.9998,1.0000]>\right\}\end{array}$ \\
\hline$m=9, n=7$ & $\begin{array}{l}\left\{<x_{1},[0.0000,0.0000],[0.9850,0.9987]>,<x_{2},[0.0000,0.0001],[0.9946,1.0000]>\right. \\
\left.<x_{3},[0.0001,0.0007],[0.9987,0.9998]>,<x_{4},[0.0007,0.0038],[0.9998,1.0000]>\right\}\end{array}$ \\
\hline$m=9, n=8$ & $\begin{aligned}\{ & <x_{1},[0.0000,0.0000],[0.9869,0.9989]>,<x_{2},[0.0000,0.0001],[0.9953,1.0000]> \\
& \left.<x_{3},[0.0001,0.0007],[0.9989,0.9999]>,<x_{4},[0.0007,0.0036],[0.9999,1.0000]>\right\}\end{aligned}$ \\
\hline$m=9, n=9$ & $\begin{aligned}\{ & <x_{1},[0.0000,0.0000],[0.9884,0.9990]>,<x_{2},[0.0000,0.0001],[0.9958,1.0000]> \\
& \left.<x_{3},[0.0001,0.0007],[0.9990,0.9999]>,<x_{4},[0.0007,0.0034],[0.9999,1.0000]>\right\}\end{aligned}$ \\
\hline$m=9, n=10$ & $\begin{aligned}\{ & <x_{1},[0.0000,0.0000],[0.9895,0.9991]>,<x_{2},[0.0000,0.0001],[0.9962,1.0000]> \\
& \left.<x_{3},[0.0001,0.0006],[0.9991,0.9999]>,<x_{4},[0.0006,0.0032],[0.9999,1.0000]>\right\}\end{aligned}$ \\
\hline$m=10, n=1$ & $\begin{aligned}\{ & <x_{1},[0.0000,0.0000],[0.9083,0.9942]>,<x_{2},[0.0000,0.0001],[0.9714,1.0000]> \\
& \left.<x_{3},[0.0001,0.0010],[0.9942,0.9994]>,<x_{4},[0.0010,0.0060],[0.9994,1.0000]>\right\}\end{aligned}$ \\
\hline
\end{tabular}


Table 2. The results of operation $P^{(m, n)}$ when $m$ and $n$ are with different values (continued).

\begin{tabular}{|c|c|}
\hline$m, n$ & Results \\
\hline$m=10, n=2$ & $\begin{aligned}\{ & <x_{1},[0.0000,0.0000],[0.9553,0.9971]>,<x_{2},[0.0000,0.0001],[0.9858,1.0000]> \\
& \left.<x_{3},[0.0001,0.0007],[0.9971,0.9997]>,<x_{4},[0.0007,0.0043],[0.9997,1.0000]>\right\}\end{aligned}$ \\
\hline$m=10, n=3$ & $\begin{array}{r}\left\{<x_{1},[0.0000,0.0000],[0.9704,0.9981]>,<x_{2},[0.0000,0.0001],[0.9906,1.0000]>\right. \\
\left.<x_{3},[0.0001,0.0006],[0.9981,0.9998]>,<x_{4},[0.0006,0.0035],[0.9998,1.0000]>\right\}\end{array}$ \\
\hline$m=10, n=4$ & $\begin{aligned}\{ & <x_{1},[0.0000,0.0000],[0.9779,0.9986]>,<x_{2},[0.0000,0.0001],[0.9929,1.0000]> \\
& \left.<x_{3},[0.0001,0.0005],[0.9986,0.9999]>,<x_{4},[0.0005,0.0030],[0.9999,1.0000]>\right\}\end{aligned}$ \\
\hline$m=10, n=5$ & $\begin{aligned}\{ & <x_{1},[0.0000,0.0000],[0.9824,0.9988]>,<x_{2},[0.0000,0.0000],[0.9944,1.0000]> \\
& \left.<x_{3},[0.0000,0.0004],[0.9988,0.9999]>,<x_{4},[0.0004,0.0027],[0.9999,1.0000]>\right\}\end{aligned}$ \\
\hline$m=10, n=6$ & $\begin{array}{r}\left\{<x_{1},[0.0000,0.0000],[0.9853,0.9990]>,<x_{2},[0.0000,0.0000],[0.9953,1.0000]>\right. \\
\left.<x_{3},[0.0000,0.0004],[0.9990,0.9999]>,<x_{4},[0.0004,0.0025],[0.9999,1.0000]>\right\}\end{array}$ \\
\hline$m=10, n=7$ & $\begin{array}{l}\left\{<x_{1},[0.0000,0.0000],[0.9874,0.9992]>,<x_{2},[0.0000,0.0000],[0.9960,1.0000]>\right. \\
\left.<x_{3},[0.0000,0.0004],[0.9992,0.9999]>,<x_{4},[0.0004,0.0023],[0.9999,1.0000]>\right\}\end{array}$ \\
\hline$m=10, n=8$ & $\begin{array}{l}\left\{<x_{1},[0.0000,0.0000],[0.9890,0.9993]>,<x_{2},[0.0000,0.0000],[0.9965,1.0000]>\right. \\
\left.<x_{3},[0.0000,0.0003],[0.9993,0.9999]>,<x_{4},[0.0003,0.0021],[0.9999,1.0000]>\right\}\end{array}$ \\
\hline$m=10, n=9$ & $\begin{aligned}\{ & <x_{1},[0.0000,0.0000],[0.9902,0.9994]>,<x_{2},[0.0000,0.0000],[0.9969,1.0000]> \\
& \left.<x_{3},[0.0000,0.0003],[0.9994,0.9999]>,<x_{4},[0.0003,0.0020],[0.9999,1.0000]>\right\}\end{aligned}$ \\
\hline$m=10, n=10$ & $\begin{aligned}\{ & <x_{1},[0.0000,0.0000],[0.9912,0.9994]>,<x_{2},[0.0000,0.0000],[0.9972,1.0000]> \\
& \left.<x_{3},[0.0000,0.0003],[0.9994,0.9999]>,<x_{4},[0.0003,0.0019],[0.9999,1.0000]>\right\}\end{aligned}$ \\
\hline
\end{tabular}

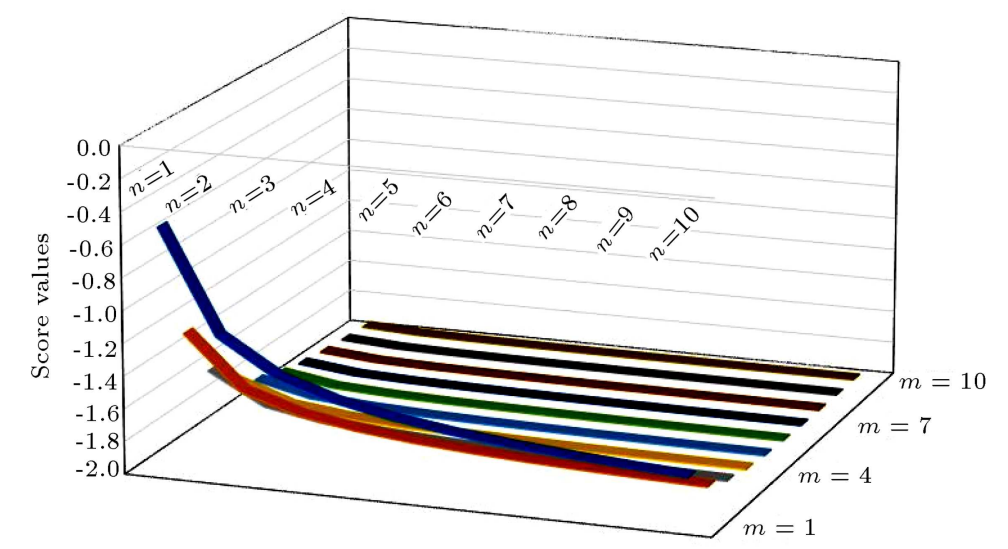

\begin{tabular}{|c|c|c|c|c|c|c|c|c|c|c|}
\hline & $n=1$ & $n=2$ & $n=3$ & $n=4$ & $n=5$ & $n=6$ & $n=7$ & $n=8$ & $n=9$ & $n=10$ \\
\hline$m=1$ & -0.5 & -1.1367 & -1.3492 & -1.4623 & -1.5343 & -1.5849 & -1.6228 & -1.6525 & -1.6765 & -1.6964 \\
\hline$m=2$ & -1.2443 & -1.5702 & -1.6807 & -1.7389 & -1.7757 & -1.8013 & -1.8204 & -1.8352 & -1.8472 & -1.857 \\
\hline$m=3$ & -1.5737 & -1.7625 & -1.8263 & -1.8596 & -1.8804 & -1.8948 & -1.9055 & -1.9137 & -1.9203 & -1.9257 \\
\hline$m=4$ & -1.7393 & -1.8579 & -1.8977 & -1.9183 & -1.931 & -1.9398 & -1.9462 & -1.9512 & -1.9551 & -1.9583 \\
\hline$m=5$ & -1.8302 & -1.9094 & -1.9358 & -1.9493 & -1.9576 & -1.9632 & -1.9674 & -1.9705 & -1.973 & -1.9751 \\
\hline$m=6$ & -1.8838 & -1.9391 & -1.9574 & 1.9667 & -1.9724 & -1.9763 & -1.9791 & -1.9812 & -1.9829 & -1.9842 \\
\hline$m=7$ & -1.9173 & -1.9573 & -1.9705 & -1.9771 & -1.9812 & -1.9839 & -1.9859 & -1.9874 & -1.9886 & -1.9895 \\
\hline $\mathbf{a}=8$ & -1.9392 & -1.9689 & -1.9787 & -1.9837 & -1.9867 & -1.9887 & -1.9901 & -1.9912 & -1.9921 & -1.9927 \\
\hline$m=9$ & -1.9541 & -1.9768 & -1.9842 & -1.988 & -1.9902 & -1.9917 & -1.9928 & -1.9936 & -1.9943 & -1.9948 \\
\hline$m=10$ & -1.9647 & -1.9822 & -1.988 & -1.9909 & -1.9926 & -1.9938 & -1.9946 & -1.9952 & -1.9957 & -1.9961 \\
\hline
\end{tabular}

Figure 2. The score function with taking different $m$ and $n$. 
Table 3. Comparison of the different operators.

\begin{tabular}{ccccccc}
\hline Operations & Examples & $\begin{array}{c}\text { Single } \\
\text { operation }\end{array}$ & $\begin{array}{c}\text { Binary } \\
\text { operation }\end{array}$ & $\begin{array}{c}\text { Adjustable } \\
\text { parameter }\end{array}$ & $\begin{array}{c}\text { Easy to } \\
\text { calculate }\end{array}$ & Aggregation \\
\hline$\cup[21]$ & $P \cup Q$ & No & Yes & No & Yes & No \\
$\cap[21]$ & $P \cap Q$ & No & Yes & No & Yes & No \\
$\oplus[21]$ & $P \oplus Q$ & No & Yes & No & No & Yes \\
$\otimes[21]$ & $P \otimes Q$ & No & Yes & No & No & Yes \\
$\lambda[21]$ & $P^{\lambda}$ or $\lambda P$ & Yes & No & No & No & No \\
$\subseteq[21]$ & $P \subseteq Q$ & No & Yes & No & Yes & No \\
$c[21]$ & $P^{c}$ & Yes & No & No & Yes & No \\
\hline$\square$ & $\square P$ & Yes & No & No & Yes & No \\
$\diamond$ & $\diamond P$ & Yes & No & No & No & No \\
$\mathbf{4}$ & $P \$ Q$ & No & Yes & No & No & Yes \\
$\rightarrow$ & $P \rightarrow Q$ & No & Yes & No & Yes & No \\
$\$$ & $P \$ Q$ & No & Yes & No & No & No \\
$\mathbf{4}$ & $P \$ Q$ & No & Yes & No & No & No \\
$(m, n)$ & $P \$ Q$ & No & Yes & No & No & No \\
\hline & $P(m, n)$ & Yes & No & Yes & No & No \\
\hline
\end{tabular}

\subsubsection{Comparison with some existing interval-valued} Pythagorean fuzzy operators

The proposed interval-valued Pythagorean fuzzy operators promote the development of fundamental properties of IVPFS. For the sake of clarity, several comparative analyses are conducted to demonstrate the advantages and disadvantages of the new operators with existing operators $[5,21]$. Table 3 presents further details.

\section{Novel approach to MCDM with interval-valued Pythagorean fuzzy information based on new operator}

\subsection{Problem description}

This section introduces a novel approach to MCDM under the interval-valued Pythagorean fuzzy environment. A typical MCDM problem with the intervalvalued Pythagorean fuzzy information can be described as follows. Let $A=A_{1}, A_{2}, \cdots, A_{m}$ be a set of alternatives and $C=\left\{C_{1}, C_{2}, \cdots, C_{n}\right\}$ be a set of attributes with the weight vector being $W=\left\{w_{1}, w_{2}, \cdots, w_{n}\right\}$, thereby satisfying $w_{i} \in[0,1]$ and $\sum_{j=1}^{n} w_{j}=1$. The expert is organized to decide on alternatives. For attribute $C_{j}(j=1,2, \cdots, n)$ of alternative $A_{i}$ $(i=1,2, \cdots, m)$, the decision-maker is required to use IVPFNs to express their preference information, which can be denoted as

$$
\begin{aligned}
p_{i j}= & \left(\left[\mu_{i j}^{-}, \mu_{i j}^{+}\right],\left[\nu_{i j}^{-}, \nu_{i j}^{+}\right]\right) \\
& (i=1,2, \cdots, m ; j=1,2, \cdots, n) .
\end{aligned}
$$

Therefore, an interval-valued Pythagorean fuzzy decision matrix can be obtained by $P=\left(p_{i j}\right)_{m \times n}$. A novel approach based on the interval-valued Pythagorean fuzzy operator $\boldsymbol{\phi}$ is introduced to solve this problem.

Step 1. Transform matrix $P=\left(P_{i j}\right)_{n}$ into a normalized interval-valued Pythagorean fuzzy matri $P^{\prime}=\left(p_{i j}^{\prime}\right)_{m \times n}$ by Eq. (10);

$$
\begin{aligned}
p_{i j}^{\prime} & =\left(\left[\left(\mu_{i j}^{-}\right)^{\prime},\left(\mu_{i j}^{+}\right)^{\prime}\right],\left[\left(\nu_{i j}^{-}\right)^{\prime},\left(\nu_{i j}^{+}\right)^{\prime}\right]\right) \\
& = \begin{cases}\left(\left[\mu_{i j}^{-}, \mu_{i j}^{+}\right],\left[\nu_{i j}^{-}, \nu_{i j}^{+}\right]\right), & C_{j} \text { is benefit criteria, } \\
\left(\left[\nu_{i j}^{-}, \nu_{i j}^{+}\right],\left[\mu_{i j}^{-}, \mu_{i j}^{+}\right]\right), & C_{j} \text { is cost criteria. }\end{cases}
\end{aligned}
$$

Step 2. For alternative $A_{i}$, we utilize the proposed A operators defined in Eq. (11) to aggregate all the attribute values:

$$
\begin{aligned}
& p_{i}=\boldsymbol{\prod}_{j=1}^{n} w_{j} p_{i j} \\
&= {\left[\sqrt{\frac{\sum_{j=1}^{n}\left(1-\left(1-\left(\mu_{i j}^{-}\right)^{2}\right)^{w_{j}}\right)}{n}},\right.} \\
& \sqrt{\left.\frac{\sum_{j=1}^{n}\left(1-\left(1-\left(\mu_{i j}^{+}\right)^{2}\right)^{w_{j}}\right)}{n}\right]}, \\
& {\left[\sqrt{\left.\frac{\sum_{j=1}^{n}\left(\left(\nu_{i j}^{-}\right)^{2 w_{j}}\right)}{n}, \sqrt{\frac{\sum_{j=1}^{n}\left(\left(\nu_{i j}^{+}\right)^{2 w_{j}}\right)}{n}}\right] .}\right.}
\end{aligned}
$$


Table 4. The tabular form of interval-valued Pythagorean fuzzy decision matrix in Example 5.

\begin{tabular}{cccccc}
\hline & $\boldsymbol{C}_{\mathbf{1}}$ & $\boldsymbol{C}_{\mathbf{2}}$ & $\boldsymbol{C}_{\boldsymbol{3}}$ & $\boldsymbol{C}_{\mathbf{4}}$ & $\boldsymbol{C}_{\mathbf{5}}$ \\
\hline $\boldsymbol{A}_{\mathbf{1}}$ & $([0.3,0.4],[0.3,0.4])$ & $([0.5,0.7],[0.6,0.7])$ & $([0.5,0.7],[0.3,0.7])$ & $([0.4,0.6],[0.6,0.7])$ & $([0.5,0.6],[0.6,0.7])$ \\
$\boldsymbol{A}_{\mathbf{2}}$ & $([0.5,0.6],[0.6,0.7])$ & $([0.5,0.6],[0.6,0.7])$ & $([0.5,0.6],[0.6,0.7])$ & $([0.5,0.6],[0.6,0.7])$ & $([0.5,0.6],[0.6,0.7])$ \\
$\boldsymbol{A}_{\mathbf{3}}$ & $([0.7,0.8],[0.1,0.2])$ & $([0.8,0.9],[0.1,0.2])$ & $([0.0,0.0],[0.2,0.3])$ & $([0.5,0.6],[0.1,0.2])$ & $([0.7,0.8],[0.1,0.2])$ \\
$\boldsymbol{A}_{\mathbf{4}}$ & $([0.3,0.4],[0.5,0.7])$ & $([0.5,0.6],[0.6,0.7])$ & $([0.3,0.5],[0.6,0.7])$ & $([0.4,0.6],[0.1,0.2])$ & $([0.5,0.6],[0.1,0.2])$ \\
$\boldsymbol{A}_{\mathbf{5}}$ & $([0.5,0.6],[0.6,0.7])$ & $([0.4,0.7],[0.1,0.2])$ & $([0.3,0.4],[0.6,0.7])$ & $([0.5,0.6],[0.3,0.4])$ & $([0.4,0.6],[0.1,0.2])$ \\
\hline
\end{tabular}

Therefore, we can obtain a series of overall values $p_{i}(i=1,2, \cdots, m)$ of alternatives;

Step 3. Rank $p_{i}$ based on score function $s\left(p_{i}\right)$ by Eq. (5);

Step 4. The alternative $A_{i}(i=1,2, \cdots, m)$ is ranked based the corresponding overall values.

\subsection{A case study in mine emergency decision making}

We will consider the emergency decision-making problems of mine accidents by employing the proposed decision-making algorithm based on new operator The mine explosion is one of the most hazardous dangers in mine accidents. The mine explosion enormously threatens the safety of workers and their lives and imperils the safety production of mine. Since the explosion accidents often occur unexpectedly and suddenly, it is not easy to predict the accident and have enough preparations and emergency actions ahead of time. Therefore, the emergency response plans and the simulations of the accidents are a requisite approach in disaster preparedness and appropriate responses. The high quality and feasibility of the emergency plans will directly influence the later emergency actions and affect the evolution of disasters. Consequently, the evaluation and decision of the given emergency plans with simulations is considered essential for the disaster management of mine accidents [42].

Example 5. Assume that there are five emergency plans $A=\left\{A_{1}, A_{2}, A_{3}, A_{4}, A_{5}\right\}$ to be considered for an explosion accident in the coal mine. The expert chooses decision parameters set $C=\left\{C_{1}, C_{2}, C_{3}, C_{4}\right\}$ to be the noxious gas concentration $C_{1}$ (denoted as gas), reducing casualty of current events $C_{2}$ (denoted as casualty), the smoke and the dust level $C_{3}$ (denoted as smoke), the feasibility of rescue operations $C_{4}$ (denoted as feasibility), and repairing facility damages caused by emergency $C_{5}$ (denoted as facility). Based on the general evolving principle and the characteristics of the mine accidents, we can determine that all attributes are benefit attributes. Suppose that the expert has the following prior weight set given by his/her prior experience or preference:

$$
w=\left(w_{1}, w_{2}, w_{3}, w_{4}, w_{5}\right)=(0.2,0.2,0.1,0.3,0.2) .
$$

Table 4 gives the assessment of emergency plans arising from questionnaire investigation by the expert and constructing an interval-valued Pythagorean fuzzy decision matrix with its tabular form.

In what follows, we utilize the algorithm proposed above to select emergency plans under interval-valued Pythagorean fuzzy information.

Step 1. The decision matrix does not require being normalized because all the attributes are benefit attributes;

Step 2. For the alternative, $A_{i}(i=1,2,3,4,5)$ is utilized to aggregate the attribute values. Therefore, we can obtain a set of overall values:

$$
\begin{aligned}
& p_{1}=([0.2049,0.2953],[0.8684,0.9128]), \\
& p_{2}=([0.2361,0.2916],[0.9038,0.9316]), \\
& p_{3}=([0.3223,0.3943],[0.6588,0.7406]), \\
& p_{4}=([0.1954,0.2679],[0.7906,0.8451]), \\
& p_{5}=([0.2081,0.2964],[0.7746,0.8278]) .
\end{aligned}
$$

Step 3. The scores of $p_{i}(i=1,2,3,4,5)$ are calculated based on Definition 6 to obtain:

$$
\begin{array}{ll}
s\left(p_{1}\right)=-1.2809, & s\left(p_{2}\right)=-1.3077, \\
s\left(p_{3}\right)=-0.6828, & s\left(p_{4}\right)=-1.1725, \\
s\left(p_{5}\right)=-1.0978 . &
\end{array}
$$

Therefore, the rank of the overall values is:

$$
s\left(p_{3}\right)>s\left(p_{5}\right)>s\left(p_{4}\right)>s\left(p_{1}\right)>s\left(p_{2}\right) .
$$

Step 4 . The alternative $A_{i}(i=1,2,3,4,5)$ is ranked based on $s\left(p_{i}\right)(i=1,2,3,4,5)$ to obtain $A_{3} \succ A_{5} \succ$ $A_{4} \succ A_{1} \succ A_{2}$. Therefore, $A_{3}$ is the best alternative.

\subsection{A comparison analysis}

In order to further verify the practicability of the proposed multi-attribute decision making approach based on the proposed operator under IVPFS environment, a comparison study with some existing methods $[17,32,37]$ is now conducted.

First, we give two core definitions related to interval-valued Pythagorean fuzzy aggregation operators in the following. 
Definition 12. [32] Let $p_{i}=\left(\left[\mu_{i}^{-}, \mu_{i}^{+}\right],\left[\nu_{i}^{-}, \nu_{i}^{+}\right]\right)$ $(i=1,2, \cdots, m)$ be a collection of IVPFNs. Then, the aggregated value by using an Interval-valued Pythagorean Fuzzy Weighted Average (IPFWA) operator is defined as:

$$
\begin{gathered}
\operatorname{IPFWA}\left(p_{1}, p_{2}, \cdots, p_{n}\right)=\bigoplus_{i=1}^{n} w_{i} p_{i} \\
=\left(\left[\sqrt{1-\prod_{i=1}^{n}\left(1-\left(\mu_{i}^{-}\right)^{2}\right)^{w_{i}},}\right.\right. \\
\left.\sqrt{1-\prod_{i=1}^{n}\left(1-\left(\mu_{i}^{+}\right)^{2}\right)^{w_{i}}}\right], \\
\left.\left[\prod_{i=1}^{n}\left(\nu_{i}^{-}\right)^{w_{i}}, \prod_{i=1}^{n}\left(\nu_{i}^{+}\right)^{w_{i}}\right]\right) .
\end{gathered}
$$

However, we can see that the IPFWA operator has drawbacks in some cases described as follows. Let $p_{j}$ $(j=1,2, \cdots, n)$ be a collection of IVPFNs. If there is $i$ such that $p_{i}=[1,1],[0,0]$, then, based on Eq. (12), we can have:

$$
\operatorname{IPFWA}\left(p_{1}, p_{2}, \cdots, p_{n}\right)=([1,1],[0,0]) .
$$

This result may cause counter-intuitive phenomena in MCDM. In other words, it is only determined by $p_{i}$ to make a decision, and the decision information of others can be neglected.

Moreover, based on Eq. (12), if there is an IVPFN such that $p_{i}=\left(\left[\mu_{i}^{-}, \mu_{i}^{+}\right],[0,0]\right)$, the aggregated value is:

$$
\operatorname{IPFWA}\left(p_{1}, p_{2}, \cdots, p_{n}\right)=\left(\left[\mu^{-}, \mu^{+}\right],[0,0]\right) .
$$

In other words, the non-membership degree of aggregated value must be zero. This result may cause counter-intuitive phenomena in some cases. Hence, it is unreasonable and unsuitable to apply Eq. (12) to aggregate the information in MCDM when meeting the special cases mentioned above.

Definition 13. [32] Let $p_{i}=\left(\left[\mu_{i}^{-}, \mu_{i}^{+}\right],\left[\nu_{i}^{-}, \nu_{i}^{+}\right]\right)$ $(i=1,2, \cdots, m)$ be a collection of IVPFNs, and then the aggregated value by using an Interval-valued
Pythagorean Fuzzy Weighted Geometric (IPFWG) operator is defined as:

$$
\begin{aligned}
& \operatorname{IPFWG}\left(p_{1}, p_{2}, \cdots, p_{n}\right)=\otimes_{i=1}^{n}\left(p_{i}\right)^{w_{i}} \\
&=\left(\left[\prod_{i=1}^{n}\left(\mu_{i}^{-}\right)^{w_{i}}, \prod_{i=1}^{n}\left(\mu_{i}^{+}\right)^{w_{i}}\right],\right. \\
& {\left[\sqrt{1-\prod_{i=1}^{n}\left(1-\left(\nu_{i}^{-}\right)^{2}\right)^{w_{i}}}\right.} \\
& \sqrt{\left.\left.1-\prod_{i=1}^{n}\left(1-\left(\nu_{i}^{+}\right)^{2}\right)^{w_{i}}\right]\right) .}
\end{aligned}
$$

However, we can see that the IPFWA operator also has drawbacks in some cases, described as follows. $p_{j}(j=1,2, \cdots, n)$ is a collection of IVPFNs. If there is $i$ such that $p_{i}=[0,0],[1,1]$, then, based on Eq. (13), we can have:

$$
\operatorname{IPFWA}\left(p_{1}, p_{2}, \cdots, p_{n}\right)=([0,0],[1,1]) .
$$

This result may cause counter-intuitive phenomena in MCDM. In other words, it is only determined by $p_{i}$ to make a decision, and the decision information of others can be neglected.

Moreover, based on Eq. (13), if there is an IVPFN such that $p_{i}=\left([0,0],\left[\nu_{i}^{-}, \nu_{i}^{+}\right]\right)$, the aggregated value is

$$
\operatorname{IPFWG}\left(p_{1}, p_{2}, \cdots, p_{n}\right)=\left([0,0],\left[\nu^{-}, \nu^{+}\right]\right) \text {. }
$$

In other words, the membership degree of aggregated value must be zero. This result may cause counterintuitive phenomena in some cases. Hence, it is unreasonable and unsuitable to apply Eq. (13) to aggregate the information in MCDM when meeting the special cases mentioned above.

\subsubsection{A comparison analysis from Example 5}

We take Example 5, and the interval-valued Pythagorean fuzzy decision matrix show in Table 4.

If the existing methods in Garg [32], Rahman et al. [37], Liang et al. [17], and the proposed method are applied to solve the MCDM problem in Example 5, then the results can be obtained, as shown in Table 5 .

Table 5. A comparison study with some existing methods in Example 5.

\begin{tabular}{llc}
\hline \multicolumn{1}{c}{ Method } & The final ranking & $\begin{array}{c}\text { The optimal } \\
\text { alternative }\end{array}$ \\
\hline Garg: IPFWA [32] & $A_{3} \succ A_{5} \succ A_{4} \succ A_{1} \succ A_{2}$ & $A_{3}$ \\
Garg: IPFWG [32] & $A_{5} \succ A_{4} \succ A_{1} \succ A_{2} \succ A_{3}$ & $A_{5}$ \\
Rahman et al.: IPFWG [37] & $A_{5} \succ A_{4} \succ A_{1} \succ A_{2}$ & $A_{5}$ \\
Liang et al.: IPFWA [17] & $A_{3} \succ A_{5} \succ A_{4} \succ A_{1} \succ A_{2}$ & $A_{3}$ \\
Proposed algorithm & $A_{3} \succ A_{5} \succ A_{4} \succ A_{1} \succ A_{2}$ & $A_{3}$ \\
\hline
\end{tabular}


Table 6. The tabular form of interval-valued Pythagorean fuzzy decision matrix in Example 5.

\begin{tabular}{cccccc}
\hline & $\boldsymbol{C}_{\mathbf{1}}$ & $\boldsymbol{C}_{\mathbf{2}}$ & $\boldsymbol{C}_{\boldsymbol{3}}$ & $\boldsymbol{C}_{\mathbf{4}}$ & $\boldsymbol{C}_{\mathbf{5}}$ \\
\hline $\boldsymbol{A}_{\mathbf{1}}$ & $([0.3,0.4],[0.3,0.4])$ & $([0.5,0.7],[0.6,0.7])$ & $([0.5,0.7],[0.3,0.7])$ & $([0.4,0.6],[0.6,0.7])$ & $([0.5,0.6],[0.6,0.7])$ \\
$\boldsymbol{A}_{\mathbf{2}}$ & $([0.5,0.6],[0.6,0.7])$ & $([0.5,0.6],[0.6,0.7])$ & $([0.5,0.6],[0.6,0.7])$ & $([0.5,0.6],[0.6,0.7])$ & $([0.5,0.6],[0.6,0.7])$ \\
$\boldsymbol{A}_{\mathbf{3}}$ & $([0.1,0.2],[0.1,0.2])$ & $([0.1,0.1],[0.1,0.2])$ & $([0.9,1.0],[0.0,0.0])$ & $([0.2,0.3],[0.6,0.7])$ & $([0.4,0.4],[0.1,0.2])$ \\
$\boldsymbol{A}_{\mathbf{4}}$ & $([0.8,0.9],[0.1,0.2])$ & $([0.6,0.1],[0.1,0.2])$ & $([0.7,0.8],[0.2,0.3])$ & $([0.6,0.8],[0.1,0.2])$ & $([0.7,0.9],[0.1,0.2])$ \\
$\boldsymbol{A}_{\mathbf{5}}$ & $([0.5,0.6],[0.6,0.7])$ & $([0.4,0.7],[0.1,0.2])$ & $([0.3,0.4],[0.6,0.7])$ & $([0.5,0.6],[0.3,0.4])$ & $([0.4,0.6],[0.1,0.2])$ \\
\hline
\end{tabular}

Table 7. A comparison study with some existing methods in Example 6.

\begin{tabular}{llc}
\hline \multicolumn{1}{c}{ Method } & The final ranking & $\begin{array}{c}\text { The optimal } \\
\text { alternative }\end{array}$ \\
\hline Garg: IPFWA [32] & $A_{3} \succ A_{4} \succ A_{5} \succ A_{1} \succ A_{2}$ & $A_{3}$ \\
Garg: IPFWG [32] & $A_{4} \succ A_{3} \succ A_{5} \succ A_{1} \succ A_{2}$ & $A_{4}$ \\
Rahman et al.: IPFWG [37] & $A_{4} \succ A_{3} \succ A_{5} \succ A_{1} \succ A_{2}$ & $A_{4}$ \\
Liang et al.: IPFWA [17] & $A_{3} \succ A_{4} \succ A_{5} \succ A_{1} \succ A_{2}$ & $A_{3}$ \\
Proposed algorithm & $A_{4} \succ A_{3} \succ A_{5} \succ A_{1} \succ A_{2}$ & $A_{4}$ \\
\hline
\end{tabular}

According to the above results shown in Table 5 , the ranking order of the four alternatives and optimal alternative is in agreement with the results of [17,32]. For Garg [32] (IPFWG) and Rahman et al. [37] (IPFWG), the optimal alternative is $A_{5}$, which is different from other methods, and it is unreasonable due to its drawback discussed in Definition 13.

\subsubsection{A comparison analysis from Example 6}

Example 6 is the continuation of Example 5. Suppose that the another expert has the following prior weight set given by his/her prior experience or preference:

$$
w=\left(w_{1}, w_{2}, w_{3}, w_{4}, w_{5}\right)=(0.2,0.2,0.1,0.3,0.2) .
$$

Table 6 shows the assessment of emergency plans arising from questionnaire investigation by the expert and constructing an interval-valued Pythagorean fuzzy decision matrix with its tabular form.

If the existing methods in Garg [32], Rahman et al. [37], Liang et al. [17], and the proposed method are applied to solve the MCDM problem in Example 6, then the results can be obtained, as shown in Table 7 .

According to the above results shown in Table 7 , the ranking order of the four alternatives and optimal alternative is in agreement with the results of $[32,37]$. For Garg [32] (IPFWA) and Liang et al. [17] (IPFWA), the optimal alternative is $A_{3}$, which is different from other methods, and it is unreasonable due to its drawback discussed in Definition 12.

\section{Conclusions}

This paper presented some novel interval-valued Pythagorean fuzzy operators $(\diamond, \square, \mathbf{A}, \mathbf{h}, \boldsymbol{\Psi}, \rightarrow, \$)$ and discussed their properties with some existing operators $(\cup, \cap, \oplus, \otimes)$ in detail. Meanwhile, a new decisionmaking method based on $\boldsymbol{A}$ operator was given. In the future, we will apply the proposed operators $(\diamond, \square, \boldsymbol{4}, \mathbf{s}, \boldsymbol{\Psi}, \rightarrow, \$)$ to other fuzzy environment $[26,43-$ 57 .

\section{Acknowledgements}

The authors are very grateful to the anonymous reviewers for their valuable comments and constructive suggestions that greatly improved the quality of this paper. Our work is sponsored by the National Natural Science Foundation of China (61163036) and the General Project of Shaoguan University (No. SY2016KJ11).

\section{References}

1. Atanassov, K.T. "Intuitionistic fuzzy sets", Fuzzy Sets Syst., 20(1), pp. 87-96 (1986).

2. Zadeh, L.A. "Fuzzy sets", Inform. Control., 8(3), pp. 338-356 (1965).

3. Yager, R.R. "Pythagorean fuzzy subsets", Proc. Joint IFSA World Congress NAFIPS Annual Meet., 1, Edmonton, Canada, pp. 57-61 (2013).

4. Yager, R.R. and Abbasov, A.M. "Pythagorean membership grades, complex numbers, and decision making", Int. J. Intell. Syst., 28(5), pp. 436-452 (2014).

5. Zhang, X.L. and Xu, Z.S. "Extension of TOPSIS to multiple criteria decision making with Pythagorean fuzzy sets", Int. J. Intell. Syst., 29(12), pp. 1061-1078 (2014).

6. Yang, Y., Ding, H., Chen, Z.S., and Li Y.L. "A Note on extension of TOPSIS to multiple criteria decision 
making with Pythagorean fuzzy sets", Int. J. Intell. Syst., 31(1), pp. 68-72 (2015).

7. Yager, R.R. "Pythagorean membership grades in multicriteria decision making", IEEE Trans. Fuzzy Syst., 22(4), pp. 958-965 (2014).

8. Peng, X.D. and Yang, Y. "Some results for Pythagorean fuzzy sets", Int. J. Intell. Syst., 30(11), pp. 1133-1160 (2015).

9. Garg, H. "Some methods for strategic decision-making problems with immediate probabilities in Pythagorean fuzzy environment", Int. J. Intell. Syst., 33(4), pp. 687-712 (2018).

10. Zhang, X.L. "Multicriteria Pythagorean fuzzy decision analysis: A hierarchical QUALIFLEX approach with the closeness index-based ranking methods", Inform. Sci, 330, pp. 104-124 (2016).

11. Peng, X.D. and Dai, J.G. "Approaches to Pythagorean fuzzy stochastic multi-criteria decision making based on prospect theory and regret theory with new distance measure and score function", Int. J. Intell. Syst., 32(11), pp. 991-1029 (2017).

12. Ren, P.J., Xu, Z.S., and Gou, X.J. "Pythagorean fuzzy TODIM approach to multi-criteria decision making", Appl. Soft Comput., 42, pp. 246-259 (2016).

13. Peng, X.D. and Yang, Y. "Pythagorean fuzzy choquet integral based MABAC method for multiple attribute group decision making", Int. J. Intell. Syst., 31(10), pp. 989-1020 (2016).

14. Garg, H. "A new generalized Pythagorean fuzzy information aggregation using Einstein operations and its application to decision making", Int. J. Intell. Syst., 31(9), pp. 886-920 (2016).

15. Garg, H. "Generalized Pythagorean fuzzy geometric aggregation operators using Einstein t-norm and tconorm for multicriteria decision-making process", Int. J. Intell. Syst., 32(6), pp. 597-630 (2017).

16. Zeng, S.Z., Chen, J.P., and Li, X.S. "A hybrid method for Pythagorean fuzzy multiple-criteria decision making", Int. J. Inf. Tech. Decis., 15(2), pp. 403-422 (2016).

17. Liang, W., Zhang, X.L., and Liu, M.F. "The maximizing deviation method based on interval-valued Pythagorean fuzzy weighted aggregating operator for multiple criteria group decision analysis", Discrete Dyn. Nat. Soc., 2015(1), pp. 1-15 (2015).

18. Garg, H. "Confidence levels based Pythagorean fuzzy aggregation operators and its application to decisionmaking process", Comput. Math. Organ. Theory, 23(4), pp. 546-571 (2017).

19. Ma, Z.M. and Xu, Z.S. "Symmetric Pythagorean fuzzy weighted geometric/averaging operators and their application in multicriteria decision-making problems", Int. J. Intell. Syst., 31(12), pp. 1198-1219 (2016).

20. Zeng, S.Z. "Pythagorean fuzzy multiattribute group decision making with probabilistic information and OWA approach", Int. J. Intell. Syst., 32(11), pp. 11361150 (2017).
21. Peng, X.D. and Yang, Y. "Fundamental properties of interval-valued Pythagorean fuzzy aggregation operators", Int. J. Intell. Syst., 31(5), pp. 444-487 (2016).

22. Wei, G.W. and Lu, M. "Pythagorean fuzzy maclaurin symmetric mean operators in multiple attribute decision making", Int. J. Intell. Syst., (2017) URL http://onlinelibrary.wiley.com/doi/10.1002/int.21911 /full

23. Garg, H. "A novel correlation coefficients between Pythagorean fuzzy sets and its applications to decisionmaking processes", Int. J. Intell. Syst., 31(12), pp. 1234-1252 (2016).

24. Peng, X.D., Yuan, H.Y., and Yang, Y. "Pythagorean fuzzy information measures and their applications", Int. J. Intell. Syst., 32(10), pp. 991-1029 (2017).

25. Zhang, X.L. "A novel approach based on similarity measure for Pythagorean fuzzy multiple criteria group decision making", Int. J. Intell. Syst., 31(6), pp. 593611 (2016).

26. Peng, X.D., Yang, Y., Song, J.P., and Jiang, Y. "Pythagorean fuzzy soft set and its application", Comput. Eng., 41(7), pp. 224-229 (2015).

27. Peng, X.D. and Yang, Y. "Multiple attribute group decision making methods based on Pythagorean fuzzy linguistic set", Comput. Eng. Appl., 52(23), pp. 50-54 (2016).

28. Liu, Z., Liu, P., Liu, W., and Pang, J. "Pythagorean uncertain linguistic partitioned Bonferroni mean operators and their application in multi-attribute decision making", J. Intell. Fuzzy Syst., 32(3), pp. 2779-2790 (2017).

29. Zhang, C., Li, D.Y., and Ren, R. "Pythagorean fuzzy multigranulation rough set over two universes and its applications in merger and acquisition", Int. J. Intell. Syst., 31(9), pp. 921-943 (2016).

30. Liang, D.C. and Xu, Z.S. "The new extension of TOPSIS method for multiple criteria decision making with hesitant Pythagorean fuzzy sets", Appl. Soft Comput., 60, pp. 167-179 (2017).

31. Du, Y., Hou, F., Zafar, W., Yu, Q., and Zhai, Y. "A novel method for multiattribute decision making with interval-valued Pythagorean fuzzy linguistic information", Int. J. Intell. Syst., 32(10), pp. 1085-1112 (2017).

32. Garg, H. "A novel accuracy function under intervalvalued Pythagorean fuzzy environment for solving multicriteria decision making problem", J. Intell. Fuzzy Syst., 31(1), pp. 529-540 (2016).

33. Gou, X.J., Xu, Z.S., and Ren, P.J. "The properties of continuous Pythagorean fuzzy information", Int. J. Intell. Syst., 31(5), pp. 401-424 (2016).

34. Peng, X.D. and Yuan, H.Y. "Fundamental properties of Pythagorean fuzzy aggregation operators", Fund. Inform., 147(4), pp. 415-446 (2016). 
35. Peng, X.D. and Selvachandran, G. "Pythagorean fuzzy set state of the art and future directions", Artif. Intell. Rev. (2017).

URL https://link.springer.com/article/10.1007/s10462017-9596-9

36. Garg, H. "A novel improved accuracy function for interval valued Pythagorean fuzzy sets and its applications in decision making process", Int. J. Intell. Syst., 32(12), pp. 1247-1260 (2017).

37. Rahman, K., Abdullah, S., Shakeel, M., Khan, M., and Ullah, M. "Interval-valued Pythagorean fuzzy geometric aggregation operators and their application to group decision making problem", Cogent. Math., 4, pp. 1-20 (2017).

38. Garg, H. "A new improved score function of an interval-valued Pythagorean fuzzy set based TOPSIS method", Int. J. Uncertainty Quantif., 7(5), pp. 463474 (2017).

39. Chen, T.Y. "An interval-Valued Pythagorean fuzzy outranking method with a closeness-based assignment model for multiple criteria decision making", Int. J. Intell. Syst., 33(1), pp. 126-168 (2018).

40. Atanassov, K.T. and Gargov, G. "Interval-valued intuitionistic fuzzy sets", Fuzzy Sets Syst., 31(3), pp. 343-349 (1989).

41. Xu, Z.S. "Methods for aggregating interval-valued intuitionistic fuzzy information and their application to decision making", Control Decis., 22(2), pp. 215219 (2007).

42. Hao, Z.N., Xu, Z.S., Zhao, H., and Fujita, H. "A dynamic weight determination approach based on the intuitionistic fuzzy Bayesian network and its application to emergency decision making", IEEE Trans. Fuzzy Syst., 26(4), pp. 1893-1907 (2018) .URL http://ieeexplore.ieee.org/abstract/document/ $8047330 /$

43. Alcantud, J.C.R. and Mathew, T.J. "Separable fuzzy soft sets and decision making with positive and negative attributes", Appl. Soft Comput., 59, pp. 586-595 (2017).

44. Zhan, J.M, Liu, Q., and Tutut, H. "A novel soft rough set soft rough hemirings and its multicriteria group decision making", Appl. Soft Comput., 54, pp. 393-402 (2017).

45. Wang, J., Wang, J.Q., and Zhang, H.Y. "A likelihoodbased TODIM approach based on multi-hesitant fuzzy linguistic information for evaluation in logistics outsourcing", Comput. Ind. Eng., 99, pp. 287-299 (2016).

46. Shariati, S., Abedi, M., Saedi, A., Yazdani-Chamzini, A., Tamošaitienè, J., Šaparauskas, J., and Stupak, S. "Critical factors of the application of nanotechnology in construction industry by using ANP technique under fuzzy intuitionistic environment", J. Civ. Eng. Manag., 23(7), pp. 914-925 (2017).
47. Peng, X.D. "Hesitant trapezoidal fuzzy aggregation operators based on Archimedean t-norm and t-conorm and their application in MADM with completely unknown weight information", Int. J. Uncertainty Quantif., 7(6), pp. 475-510 (2017).

48. Alcantud, J.C.R. "A novel algorithm for fuzzy soft set based decision making from multiobserver input parameter data set", Inform. Fusion, 29, pp. 142-148 (2016).

49. Garg, H. "Generalized intuitionistic fuzzy interactive geometric interaction operators using Einstein t-norm and t-conorm and their application to decision making", Comput. Ind. Eng., 101, pp. 53-69 (2016).

50. Garg, H. "A new generalized improved score function of interval-valued intuitionistic fuzzy sets and applications in expert systems", Appl. Soft Comput., 38, pp. 989-999 (2016).

51. Garg, H. "Distance and similarity measures for intuitionistic multiplicative preference relation and its applications", Int. J. Uncertainty Quantif., 7(2), pp. 117-133 (2017).

52. Peng, X.D. and Yang, Y. "Algorithms for intervalvalued fuzzy soft sets in stochastic multi-criteria decision making based on regret theory and prospect theory with combined weight", Appl. Soft Comput., 54, pp. 415-430 (2017).

53. Peng, X.D. and Liu, C. "Algorithms for neutrosophic soft decision making based on EDAS, new similarity measure and level soft set", J. Intell. Fuzzy Syst., 32(1), pp. 955-968 (2017).

54. Zhan, J.M., Ali, M., and Mehmood, N. "On a novel uncertain soft set model Z-soft fuzzy rough set model and corresponding decision making methods", Appl. Soft Comput., 56, pp. 446-457 (2017).

55. Ma, X.L., Liu, Q., and Zhan, J.M. "A survey of decision making methods based on certain hybrid soft set models", Artif. Intell. Rev., 47(4), pp. 507-530 (2017).

56. Garg, H. and Kumar, K. "A novel correlation coefficient of intuitionistic fuzzy sets based on the connection number of set pair analysis and its application", Sci. Iran., 25(4), pp. 2373-2388 (2018). URL http://scientiairanica.sharif.edu/article_4454.html

57. Garg, H., Agarwal, N., and Tripathi, A. "Some improved interactive aggregation operators under interval-valued intuitionistic fuzzy environment and its application to decision making process", Sci. Iran., 24(5), pp. 2581-2604 (2017).

\section{Biography}

Xindong Peng works in the School of Information Science and Engineering, Shaoguan University as an Assistant Professor. More than 20 SCI-indexed papers (2 Highly Cited Papers) have been published in high-quality international journals including "Applied Soft Computing", "Applied Mathematical Modelling", 
"International Journal of Intelligent Systems", "Artificial Intelligence Review". As a Reviewer of "IEEE Transactions on Fuzzy Systems", "Knowledge-Based Systems", "International Journal of Fuzzy Systems", "Iranian Journal of Fuzzy Systems", "Scientia Iranica", "Journal of Engineering Manufacture", "Journal of Intelligent and Fuzzy Systems", "FILOMAT",
"Computational and Applied Mathematics", "International Journal for Uncertainty Quantification", "Kybernetes", "Fundamenta Informaticae", "Artificial Intelligence Review". His current research interests include neutrosophic set, soft computing, multi-criteria decision making, pattern recognitions, soft set theory, and Pythagorean fuzzy set. 